\title{
FIVE-YEAR WILKINSON MICROWAVE ANISOTROPY PROBE* OBSERVATIONS: SOURCE CATALOG
}

\author{
E. L. Wright ${ }^{1}$, X. Chen $^{1}$, N. Odegard ${ }^{2}$, C. L. BennetT ${ }^{3}$, R. S. Hill ${ }^{2}$, G. Hinshaw $^{4}$, N. JarosiK ${ }^{5}$, E. Komatsu ${ }^{6}$, M. R. Nolta ${ }^{7}$, \\ L. Page ${ }^{5}$, D. N. Spergel ${ }^{8,9}$, J. L. Weiland ${ }^{2}$, E. Wollack ${ }^{4}$, J. DunKley ${ }^{5,8,10}$, B. Gold ${ }^{3}$, M. Halpern ${ }^{11}$, A. Kogut ${ }^{4}$, \\ D. LARSON ${ }^{3}$, M. LimON ${ }^{12}$, S. S. MeYer ${ }^{13}$, AND G. S. TuCKer ${ }^{14}$ \\ ${ }^{1}$ UCLA Physics \& Astronomy, P.O. Box 951547, Los Angeles, CA 90095-1547, USA; wright@astro.ucla.edu \\ ${ }^{2}$ Adnet Systems, Inc., 7515 Mission Dr., Suite A100, Lanham, MD 20706, USA \\ ${ }^{3}$ Department of Physics \& Astronomy, The Johns Hopkins University, 3400 N. Charles St., Baltimore, MD 21218-2686, USA \\ ${ }^{4}$ Code 665, NASA/Goddard Space Flight Center, Greenbelt, MD 20771, USA \\ ${ }^{5}$ Department of Physics, Jadwin Hall, Princeton University, Princeton, NJ 08544-0708, USA \\ ${ }^{6}$ Department of Astronomy, University of Texas, Austin, 2511 Speedway, RLM 15.306, Austin, TX 78712, USA \\ ${ }^{7}$ Canadian Institute for Theoretical Astrophysics, University of Toronto, 60 St. George St., Toronto, ON M5S 3H8, Canada \\ ${ }^{8}$ Department of Astrophysical Sciences, Peyton Hall, Princeton University, Princeton, NJ 08544-1001, USA \\ ${ }^{9}$ Princeton Center for Theoretical Physics, Princeton University, Princeton, NJ 08544, USA \\ ${ }^{10}$ Astrophysics, University of Oxford, Keble Road, Oxford, OX1 3RH, UK \\ ${ }^{11}$ Department of Physics and Astronomy, University of British Columbia, Vancouver, BC V6T 1Z1, Canada \\ 12 Columbia Astrophysics Laboratory, 550 W. 120th St., Mail Code 5247, New York, NY 10027-6902, USA \\ ${ }^{13}$ Departments of Astrophysics and Physics, KICP and EFI, University of Chicago, Chicago, IL 60637, USA \\ ${ }^{14}$ Department of Physics, Brown University, 182 Hope St., Providence, RI 02912-1843, USA \\ Received 2008 March 4; accepted 2008 June 10; published 2009 February 11
}

\begin{abstract}
We present the list of point sources found in the Wilkinson Microwave Anisotropy Probe (WMAP) five-year maps. The technique used in the first-year and three-year analyses now finds 390 point sources, and the fiveyear source catalog is complete for regions of the sky away from the Galactic plane to a 2 Jy limit, with SNR $>4.7$ in all bands in the least covered parts of the sky. The noise at high frequencies is still mainly radiometer noise, but at low frequencies the cosmic microwave background (CMB) anisotropy is the largest uncertainty. A separate search of CMB-free V-W maps finds 99 sources of which all but one can be identified with known radio sources. The sources seen by WMAP are not strongly polarized. Many of the WMAP sources show significant variability from year to year, with more than a 2:1 range between the minimum and maximum fluxes.
\end{abstract}

Key words: catalogs - cosmic microwave background - quasars: general - radio continuum: galaxies - surveys

\section{INTRODUCTION}

The Wilkinson Microwave Anisotropy Probe (WMAP; Bennett et al. 2003a) is a Medium-class Explorer (MIDEX) mission designed to study cosmology by producing full-sky maps of the cosmic microwave background (CMB) anisotropy. $W M A P$ has measured the angular power spectrum of the $\mathrm{CMB}$ anisotropy over $10^{3}$ different values of the spherical harmonic index $\ell$. All of these data can be adequately fitted by a simple six parameter $\Lambda \mathrm{CDM}$ model, and this model can also fit other datasets (Spergel et al. 2007). A determination of the interference from foreground sources is an essential part of the analysis of CMB data (Nolta et al. 2009). The most important foreground at small angular scales is due to extragalactic flat-spectrum radio sources. Sources are found by searching the maps for bright spots that approximate the beam profile, but due to the limited angular resolution of WMAP it is possible to confuse positive $\mathrm{CMB}$ excursions with point sources. Nonetheless, WMAP provides the only all-sky survey of the millimeter-wave sky so its point source catalogs are valuable for the study of flat-spectrum radio sources. In addition, the WMAP point source catalog is used to mask out contaminated spots in the high Galactic latitude sky used for cosmological analyses. 208 point sources were found in a search of the first year of WMAP observations (Bennett et al. 2003b). A search for point sources in the threeyear WMAP data found 323 sources (Hinshaw et al. 2007). In

* WMAP is the result of a partnership between Princeton University and NASA's Goddard Space Flight Center. Scientific guidance is provided by the WMAP Science Team. this paper we report on 390 point sources found in the WMAP five-year maps.

The signal-to-noise ratio on point sources found in WMAP depends on the the sensitivity in Janskies per pixel and the number of pixels that can be averaged to estimate the flux. Since $W M A P$ was designed to give approximately equal sensitivity in each band, and the conversion factor from Janskies to Rayleigh-Jeans brightness temperature in Kelvins within a constant pixel size is determined by the illuminated area of the telescope, the sensitivity in Janskies per pixel is fairly constant. The $\Gamma_{f f}$ factors tabulated by Hill et al. (2009) give the peak temperature expected for a 1 Jansky source as 262.7, $211.9,219.6,210.1$, and $179.2 \mu \mathrm{K}$ for the $\mathrm{K}$ through $\mathrm{W}$ bands of WMAP. But the number of pixels that can be averaged to estimate the flux is proportional to the wavelength squared, so the overall radiometer noise contribution to the point source flux uncertainty is approximately proportional to the frequency. $W M A P$ actually illuminates different fractions of the primary mirror in different bands, and does not have exactly the same sensitivity in Kelvins per pixel in each band, so the actual radiometer noise contributions to point source flux estimates are $0.067,0.11,0.13,0.23$, and $0.40 \mathrm{Jy}$ divided by the square root of the number of years of observations for sources on the ecliptic where the coverage is smallest. The anisotropy of the CMB itself is also a source of noise that does not integrate down with more years of observation. Using the point-source flux estimating filters on simulated noise-free CMB maps generated using the parameters in Spergel et al. (2007) gives $1 \sigma$ flux noises of $0.27,0.41,0.36,0.27$, and $0.14 \mathrm{Jy}$ in the $\mathrm{K}, \mathrm{Ka}, \mathrm{Q}, V$, and 
W bands (Chen \& Wright 2008). This "CMB noise" term peaks where the beam size matches the first acoustic peak.

\section{POINT SOURCES IN INDIVIDUAL BAND MAPS}

Extragalactic point sources contaminate the WMAP anisotropy data and a few hundred of them are strong enough that they should be masked and discarded prior to undertaking any $\mathrm{CMB}$ analysis. In this section we describe a new direct search for sources in the five-year WMAP band maps. Based on this search, we update the source mask that was used in the five-year analysis.

In the three-year analysis, we produced a catalog of bright point sources in the WMAP sky maps, independent of their presence in external surveys. This process has been repeated with the five-year maps as follows. We filter the weighted maps, $N_{\mathrm{obs}}^{1 / 2} T$ ( $N_{\mathrm{obs}}$ is the number of observations per pixel) in harmonic space by $b_{l} /\left(b_{l}^{2} C_{l}^{\mathrm{cmb}}+C_{l}^{\text {noise }}\right)$, (Tegmark $\&$ de Oliveira-Costa 1998; Refregier et al. 2000), where $b_{l}$ is the transfer function of the WMAP beam response (Page et al. 2003; Jarosik et al. 2007; Hill et al. 2009), $C_{l}^{\mathrm{cmb}}$ is the CMB angular power spectrum, and $C_{l}^{\text {noise }}$ is the noise power. Note that the CMB angular power spectrum used in this filtering has been updated to match the parameters from the WMAP three-year analysis, and that the importance of the noise power spectrum goes down as one over the number of years of data. Peaks that are $>5 \sigma$ in the filtered map in any band are fit in the unfiltered maps for all bands to a Gaussian profile plus a planar baseline. The Gaussian amplitude is converted to a source flux density using the conversion factors given in Hill et al. (2009). When a source is identified with $>5 \sigma$ confidence in any band, the flux densities for other bands are given if they are $>2 \sigma$ and the fit source width is within a factor of 2 of the true beam width. We cross-correlate detected sources with the GB6 (Gregory et al. 1996), PMN (Griffith et al. 1994), and Kühr et al. (1981) catalogs to identify $5 \mathrm{GHz}$ counterparts. If a $5 \mathrm{GHz}$ source is within $11^{\prime}$ of the WMAP source position (the WMAP source position uncertainty is $4^{\prime}$ ) we identify the $W M A P$ source with the $5 \mathrm{GHz}$ source and list the identification in Table 1. When more than one source lies within the cutoff radius the brightest one is assumed to be the WMAP counterpart.

The catalog of 390 sources obtained from the five-year maps is listed in Table 1. In the first-year catalog, source ID numbers were assigned on the basis of position (sorted by Galactic longitude). Now, rather than assigning new numbers to the newly detected sources, we follow Hinshaw et al. (2007) and recommend that WMAP sources be referred to by their coordinates, e.g., WMAP J0006-0622. For reference, we give the first-year source ID in Column 3 of Table 1. The 5 GHz IDs are given in the last column.

The three-year catalog contained 323 sources. Given the increased sensitivity in the five-year maps, the number of new sources detected is consistent with expectations based on differential source count models. At the same time, three sources from the first-year catalog are not present in the fiveyear list (numbers 15, 61, and 156). Source numbers 31, 96 and, 168 which were missing in the three-year list have been resurrected. Simulations of the first-year catalog suggested that it contained $5 \pm 4$ false detections, so the number of dropped first-year sources is consistent with expectations. Nine sources from the three-year catalog are missing from the fiveyear catalog: WMAP J0513-2015, 0734+5021, 1227+1124, $1231+1351,1302+4856,1309+1155,1440+4958,1556-7912$, and $1648+4114$. The sources $\mathrm{J} 1227+1124$ and $\mathrm{J} 1231+1351$

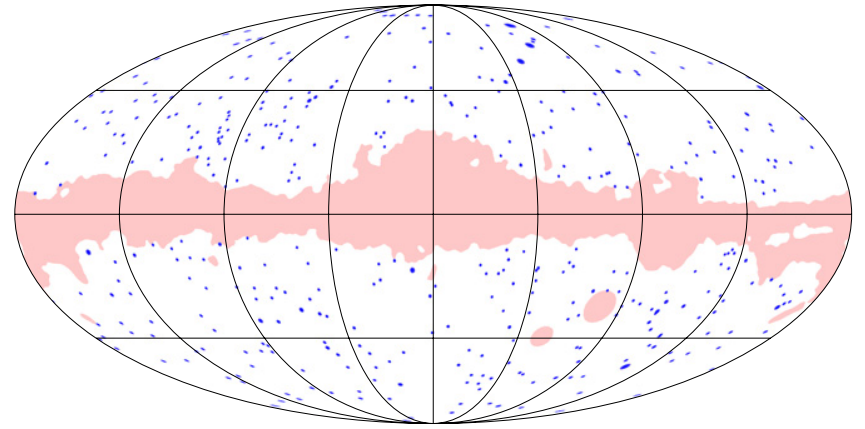

Figure 1. Map showing the location of the 390 point sources found by searching individual band maps. The shaded region shows the mask used to exclude extended foreground emission. The size of the plotted points indicates the flux of the source: the area of the dot scales like the maximum flux over the five WMAP bands plus 4 Jy. Galactic coordinates are plotted.

were spurious detections caused by sidelobes in the filtered maps around the strong source $\mathrm{J} 1230+1223$. The problem of strong source sidelobes is handled as follows in the five year analysis: after identification of each source with signal-to-noise ratio greater than 30 in a filtered map, the map is cleaned by subtracting the point-spread function scaled to the source peak. A total of six out of the 323 sources in the three-year catalog could not be identified with $5 \mathrm{GHz}$ counterparts; now 17 out of the 390 sources in the five-year catalog do not have $5 \mathrm{GHz}$ identifications. The strong source J1924-2914 is included in the five-year catalog but not in the previous catalogs because of a small change in the mask used to exclude Galactic plane and Magellanic cloud regions. Isolated mask regions with fewer than 500 contiguous HEALPix pixels at resolution 9 are no longer included in the mask (compare Figure 1 with the Kp0 mask in Figure 2 of Bennett et al. 2003b). The point source catalog mask shown in Figure 1 is available on the LAMBDA Web site, http://lambda.gsfc.nasa.gov.

Trushkin (2003) has compiled multifrequency radio spectra and high resolution radio maps of the sources in the first-year WMAP catalog. Reliable identifications are claimed for 205 of the 208 first-year sources. Of the 203 sources with optical identifications, Trushkin (2003) finds 141 quasars, 42 galaxies, or active galactic nuclei, 19 BL Lac-type objects and one planetary nebula, IC418. $40 \%$ of the sources are identified as having flat and inverted radio spectra, $13 \%$ might have GHz-peaked spectra, $8 \%$ are classical power-law sources, and $7 \%$ have a classical low frequency power law combined with a flat or inverted spectrum component (like 3C84). Trushkin (2003) suggests that the WMAP source number 116 is likely to be spurious and, for source 61 no radio component was found. Indeed, source 61 is not present in either the three-year cata$\log$ or the five-year catalog. Giommi et al. (2007) observed the 23 objects in the first WMAP sample that were not reported as X-ray sources and detected all of these objects in the 0.3$10 \mathrm{keV}$ band. They report a strong correlation between X-ray and microwave properties for these blazars.

The distribution of five-year sources on the sky is shown in Figure 1. A Kp0+LMC+SMC mask was used when finding point sources. This mask excluded $22 \%$ of the sky. The source counts in the $33 \mathrm{GHz}$ band are shown in Figure 2. The scaling of the Toffolatti et al. (1998) model has decreased from 0.66 to 0.64 . The slope of the WMAP source counts is quite close to the Euclidean $d N / d S \propto S^{-2.5}$ slope, while both the models (Toffolatti et al. 1998; de Zotti et al. 2005) and the more sensitive data (Mason et al. 2003; Cleary et al. 2005) show sub-Euclidean faint source counts. 


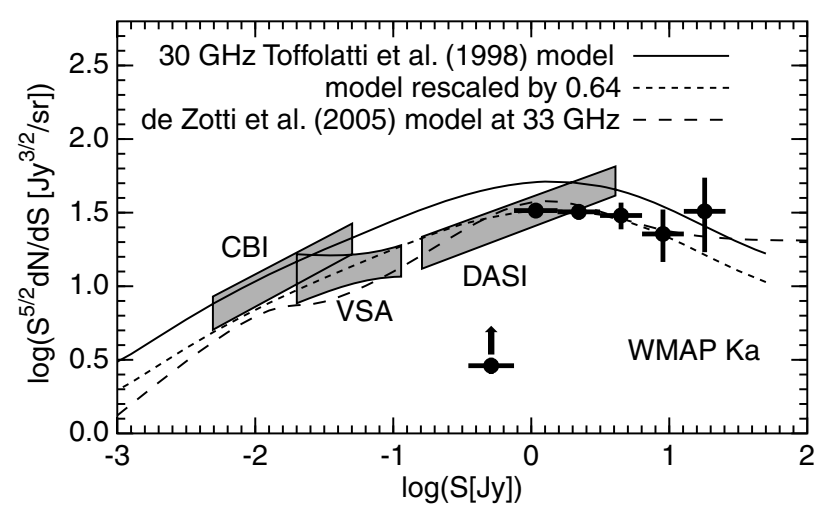

Figure 2. Differential source counts from the WMAP five-year catalog compared the Toffolatti et al. (1998) model, and to CBI counts at $31 \mathrm{GHz}$ (Mason et al. 2003), $33 \mathrm{GHz}$ VSA counts (Cleary et al. 2005), and DASI $31 \mathrm{GHz}$ counts (Kovac et al. 2002). Models from Toffolatti et al. (1998) and de Zotti et al. (2005) are shown as well. Error bars for WMAP are statistical only. The WMAP catalog in the 0.35 to $0.75 \mathrm{Jy}$ bin is quite incomplete, leading to the low data point with the upward arrow on the plot.

The spectral indices of the sources are clustered near a flat spectrum, $\alpha=0$ in $F_{v} \propto v^{\alpha}$. A histogram of the measured $\alpha$ 's is shown in Figure 3. The smooth curve is a Gaussian with a mean of $\langle\alpha\rangle=-0.09$ and $\sigma=0.28$. This $\sigma$ includes measurement errors and is thus an upper limit on the true dispersion of spectral indices. Assuming for simplicity that the underlying distributions of spectral indices is a Gaussian with standard deviation $\sigma_{\circ}$, then the intrinsic dispersion that gives $\chi^{2}$ per degree of freedom equal to unity is $\sigma_{\circ}=0.176$ and the weighted mean $\langle\alpha\rangle=-0.09$.

\subsection{Analysis of Simulated Maps}

The point-source analysis was repeated on simulated maps constructed with point sources, CMB fluctuations, and radiometer noise. $10^{6}$ sources were sampled from a power law $N(>S)$ distribution at $30 \mathrm{GHz}$. This distribution was matched to the de Zotti et al. (2005) source count model. Spectral indices were then chosen from a Gaussian with mean -0.09 and standard deviation 0.176, and the fluxes were scaled to the five WMAP band centers. For each source, the appropriate temperature in each band was then added to a randomly chosen HEALPix pixel at resolution 11 (a total of $12 \times 4^{11}$ pixels). These point source maps, one for each band, were then smoothed with the beam window function and converted to a resolution 9 map, and added to a simulated CMB plus radiometer noise maps. The point-source detection process was then applied to these simulated maps, yielding 363 point sources. Of these, only six were spurious. The recovered $N(>S)$ agreed with the simulation input for fluxes $>1 \mathrm{Jy}$, but fell well below the input at lower fluxes. Since sources with fluxes $<1 \mathrm{Jy}$ are unlikely to be detected, the ones that are detected tend to have "benefited" from a positive noise or CMB fluctuation, leading to a bias at low fluxes (Eddington 1913). The mean ratio of the derived flux to the input flux in bands $\mathrm{K}-\mathrm{V}$ is within $5 \%$ of unity for fluxes $>1 \mathrm{Jy}$, but then increases by $10 \%-20 \%$ or more for fluxes $<1 \mathrm{Jy}$. In the $\mathrm{W}$ band the measured flux is about $10 \%$ below the input flux for fluxes $>2 \mathrm{Jy}$, and rises to $>20 \%$ above the input flux for fluxes $<1 \mathrm{Jy}$. The bias in the W-band flux for high fluxes could be due to the Gaussian approximation used in flux fitting. The deviation of the mean measured spectral index from the input spectral index is about -0.02 for Q-band fluxes $>2 \mathrm{Jy}$, but rises to +0.04 at $1 \mathrm{Jy}$ and is higher than +0.10 for fluxes less than $1 \mathrm{Jy}$. We conclude that the fluxes and spectral indices are

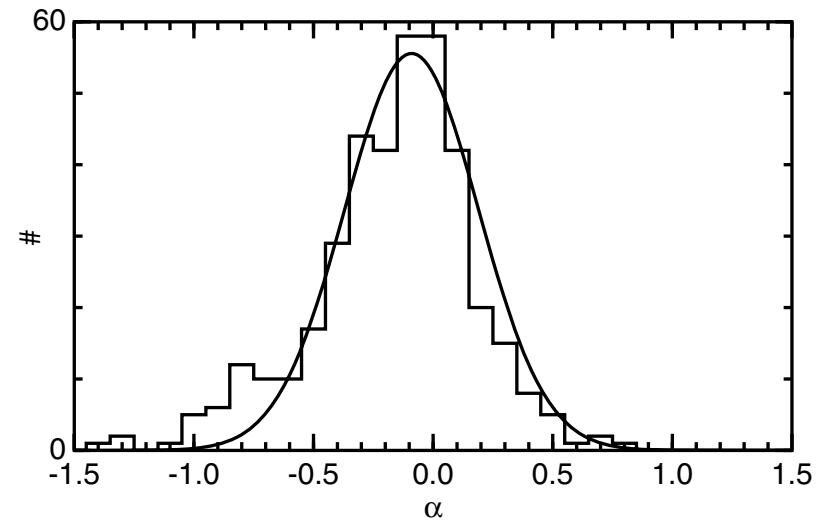

Figure 3. Histogram of the spectral indices of WMAP sources in the five-year maps. The smooth curve is a Gaussian with a mean of -0.09 and a standard deviation of 0.28 , normalized to the total number of sources.

reliable for fluxes $>2 \mathrm{Jy}$, but small biases are present for fluxes $\lesssim 1 \mathrm{Jy}$. The source counts should be reliable for fluxes $\gtrsim 1 \mathrm{Jy}$.

\section{POINT SOURCES IN CMB-FREE ILC MAPS}

The number of sources detected by WMAP as a function of integration times varied as $N \propto t_{\text {int }}^{0.4}$ between the one-year and the three-year catalogs, but slowed slightly to $\propto t_{\text {int }}^{0.37}$ between the three-year and the five-year maps. This could be due to the "noise" from the CMB, which does not integrate down with increased observing time. An approach to circumvent this noise term has been developed by Chen \& Wright (2008). It involves forming internal linear combination (ILC) maps from the WMAP bands, but unlike the normal ILC maps which preserve the $\mathrm{CMB}$ and suppress foregrounds, these ILC maps are designed to suppress the CMB. Applying this technique to the WMAP V and W bands alone, Chen \& Wright (2008) found 31 sources in the one-year maps and 64 sources in the three-year maps. This gives $N \propto t_{\text {int }}^{0.66}$ indicating that the ILC technique improves rapidly with increased observing time.

We have applied this ILC V-W technique to the five-year maps and there are 99 sources detected in the region with $|b|>10^{\circ}$. These are listed in Table 2. Among them, 64 are in the WMAP five-year source catalog, 17 can be identified with sources in NED based on continuity of spectral energy distributions, 17 are in complex Galactic emission regions, leaving only one source at $09^{\mathrm{h}} 21^{\mathrm{m}} 28^{\mathrm{s}},+7^{\circ} 24^{\prime} 22^{\prime \prime}$ without any identification. The $\mathrm{V}-\mathrm{W}$ technique can find sources sitting in negative peaks of the CMB where the standard flux finding technique returns an insignificant or even negative flux. V-band fluxes for these sources have been estimated by multiplying the value of the $\mathrm{V}-\mathrm{W}$ map in $\mathrm{mK}$, tabulated in Table 2, by the median conversion factor derived from the sources identified in Table 1. This factor is $6.28 \mathrm{Jy}$ per $\mathrm{mK}$. Of the 99 sources in Table 2, 12 are in the source list by Nie \& Zhang (2007) using the cross-correlation detection method, eight are in the new detections of the nonblind catalog by López-Caniego et al. (2007), 27 are in the AT20G Bright Source Sample (Massardi et al. 2007), and 70 are in the CRATES catalog (Healey et al. 2007).

The number of sources found by the ILC V-W technique continues to increase fairly quickly with increased integration time, going like $t^{0.72}$ from one year to five years. For Euclidean source counts the expected scaling is $t^{0.75}$. 
Table 1

WMAP Source Catalog

\begin{tabular}{|c|c|c|c|c|c|c|c|c|c|c|}
\hline R.A. [hms] & Decl. $[\mathrm{dm}]$ & ID & $\mathrm{K}[\mathrm{Jy}]$ & $\mathrm{Ka}[\mathrm{Jy}]$ & $\mathrm{Q}[\mathrm{Jy}]$ & $\mathrm{V}[\mathrm{Jy}]$ & $\mathrm{W}[\mathrm{Jy}]$ & $\alpha$ & $5 \mathrm{GHz}$ ID & \\
\hline 000320 & -4752 & & 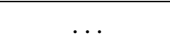 & $0.7 \pm 0.06$ & $0.7 \pm 0.09$ & $0.4 \pm 0.1$ & $\ldots$ & $-0.7 \pm 1$ & & \\
\hline 000606 & -0623 & 060 & $2.3 \pm 0.06$ & $2.3 \pm 0.1$ & $2.3 \pm 0.1$ & $2.0 \pm 0.2$ & $\ldots$ & $-0.1 \pm 0.2$ & PMN J0006-0623 & $\mathrm{v}$ \\
\hline 001037 & 1101 & & $1.1 \pm 0.07$ & $1.2 \pm 0.1$ & $1.2 \pm 0.1$ & $1.0 \pm 0.2$ & $1.6 \pm 0.5$ & $0.1 \pm 0.3$ & GB6 J0010+1058 & $\mathrm{v}$ \\
\hline 001253 & -3952 & 202 & $1.3 \pm 0.04$ & $1.3 \pm 0.08$ & $1.0 \pm 0.09$ & $1.3 \pm 0.2$ & $0.8 \pm 0.2$ & $-0.2 \pm 0.2$ & PMN J0013-3954 & \\
\hline 001918 & 2603 & & $1.0 \pm 0.06$ & $0.7 \pm 0.1$ & $0.8 \pm 0.1$ & $0.5 \pm 0.2$ & $1.4 \pm 0.3$ & $0.0 \pm 0.3$ & GB6 J0019+2602 & \\
\hline 001940 & 2020 & & $1.0 \pm 0.06$ & $1.1 \pm 0.08$ & $0.9 \pm 0.09$ & $1.3 \pm 0.2$ & $\ldots$ & $0.1 \pm 0.3$ & GB6 J0019+2021 & \\
\hline 002522 & -2602 & & $0.9 \pm 0.05$ & $0.7 \pm 0.09$ & $0.5 \pm 0.08$ & & $\ldots$ & $-0.8 \pm 0.6$ & PMN J0025-2602 & a \\
\hline 002607 & -3510 & & $1.1 \pm 0.07$ & $1.1 \pm 0.09$ & $1.4 \pm 0.1$ & $1.0 \pm 0.2$ & $\ldots$ & $0.2 \pm 0.3$ & PMN J0026-3512 & $\mathrm{v}$ \\
\hline 002934 & 0554 & & $1.1 \pm 0.06$ & $1.3 \pm 0.09$ & $1.0 \pm 0.09$ & $0.7 \pm 0.2$ & $\ldots$ & $-0.1 \pm 0.3$ & GB6 J0029+0554B & a \\
\hline 003814 & -2459 & & $0.7 \pm 0.06$ & $0.8 \pm 0.1$ & $0.6 \pm 0.1$ & $1.1 \pm 0.3$ & $\ldots$ & $0.3 \pm 0.5$ & PMN J0038-2459 & \\
\hline 004312 & 5208 & & $1.0 \pm 0.04$ & $0.6 \pm 0.07$ & $0.5 \pm 0.08$ & $0.5 \pm 0.1$ & $\ldots$ & $-1.0 \pm 0.4$ & GB6 J0043+5203 & \\
\hline 004719 & -2514 & 062 & $1.1 \pm 0.06$ & $0.9 \pm 0.1$ & $1.1 \pm 0.1$ & $1.0 \pm 0.2$ & $0.9 \pm 0.2$ & $-0.1 \pm 0.3$ & PMN J0047-2517 & \\
\hline 004950 & -5739 & 179 & $1.4 \pm 0.05$ & $1.4 \pm 0.07$ & $1.2 \pm 0.07$ & $1.2 \pm 0.2$ & $0.8 \pm 0.3$ & $-0.2 \pm 0.2$ & PMN J0050-5738 & $\mathrm{v}$ \\
\hline 005048 & -4224 & & $1.3 \pm 0.03$ & $1.3 \pm 0.06$ & $1.2 \pm 0.06$ & $0.7 \pm 0.1$ & $0.8 \pm 0.2$ & $-0.2 \pm 0.2$ & PMN J0051-4226 & $\mathrm{v}$ \\
\hline 005049 & -0649 & & $1.1 \pm 0.06$ & $1.1 \pm 0.09$ & $0.7 \pm 0.1$ & $1.3 \pm 0.2$ & $1.2 \pm 0.5$ & $-0.0 \pm 0.3$ & PMN J0051-0650 & \\
\hline 005102 & -0927 & 077 & $1.0 \pm 0.06$ & $1.0 \pm 0.08$ & $0.8 \pm 0.09$ & $1.1 \pm 0.2$ & $\ldots$ & $-0.1 \pm 0.3$ & PMN J0050-0928 & \\
\hline 010008 & -5654 & & $0.5 \pm 0.04$ & $0.7 \pm 0.08$ & $0.8 \pm 0.08$ & $0.5 \pm 0.1$ & $\ldots$ & $0.3 \pm 0.4$ & 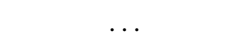 & \\
\hline 010643 & -4035 & 171 & $2.2 \pm 0.04$ & $2.4 \pm 0.07$ & $2.2 \pm 0.09$ & $2.0 \pm 0.2$ & $1.5 \pm 0.3$ & $-0.0 \pm 0.1$ & PMN J0106-4034 & $\mathrm{v}$ \\
\hline 010830 & 1319 & 079 & $1.4 \pm 0.06$ & $1.1 \pm 0.1$ & $0.8 \pm 0.2$ & & $\ldots$ & $-0.8 \pm 0.6$ & GB6 J0108+1319 & \\
\hline 010843 & 0135 & 081 & $1.9 \pm 0.06$ & $1.9 \pm 0.08$ & $1.7 \pm 0.1$ & $1.5 \pm 0.2$ & $\ldots$ & $-0.1 \pm 0.2$ & GB6 J0108+0135 & $\mathrm{a}, \mathrm{V}$ \\
\hline 011521 & -0129 & & $0.9 \pm 0.05$ & $1.2 \pm 0.08$ & $1.0 \pm 0.09$ & $1.1 \pm 0.1$ & $\ldots$ & $0.2 \pm 0.3$ & PMN J0115-0127 & \\
\hline 011618 & -1137 & & $1.3 \pm 0.07$ & $1.0 \pm 0.1$ & $1.0 \pm 0.1$ & $1.5 \pm 0.3$ & $\ldots$ & $-0.1 \pm 0.4$ & PMN J0116-1136 & \\
\hline 012146 & 1150 & & $1.2 \pm 0.05$ & $1.1 \pm 0.1$ & $1.2 \pm 0.1$ & $0.6 \pm 0.2$ & $\ldots$ & $-0.3 \pm 0.4$ & GB6 J0121+1149 & \\
\hline 012521 & -0010 & 086 & $1.1 \pm 0.06$ & $1.2 \pm 0.09$ & $1.1 \pm 0.1$ & $0.8 \pm 0.2$ & $\ldots$ & $-0.0 \pm 0.3$ & PMN J0125-0005 & a \\
\hline 013236 & -1653 & 097 & $1.8 \pm 0.05$ & $1.8 \pm 0.09$ & $1.8 \pm 0.1$ & $1.6 \pm 0.2$ & $1.3 \pm 0.3$ & $-0.1 \pm 0.2$ & PMN J0132-1654 & \\
\hline 013308 & -5200 & 168 & $0.8 \pm 0.05$ & $1.1 \pm 0.08$ & $0.7 \pm 0.07$ & $\ldots$ & $\ldots$ & $0.0 \pm 0.4$ & PMN J0133-5159 & \\
\hline 013326 & -3627 & & $0.6 \pm 0.06$ & $0.6 \pm 0.1$ & & $\ldots$ & $\ldots$ & $-0.3 \pm 1$ & PMN J0134-3629 & a \\
\hline 013701 & 4753 & 080 & $3.8 \pm 0.05$ & $3.8 \pm 0.09$ & $3.6 \pm 0.1$ & $3.2 \pm 0.2$ & $1.8 \pm 0.2$ & $-0.2 \pm 0.09$ & GB6 J0136+4751 & $\mathrm{v}$ \\
\hline 013737 & -2428 & & $1.3 \pm 0.06$ & $1.3 \pm 0.09$ & $1.8 \pm 0.1$ & $1.4 \pm 0.2$ & $\ldots$ & $0.4 \pm 0.3$ & PMN J0137-2430 & $\mathrm{v}$ \\
\hline 014910 & 0553 & & $1.0 \pm 0.06$ & $0.7 \pm 0.09$ & $0.8 \pm 0.1$ & & $\ldots$ & $-0.4 \pm 0.5$ & GB6 J0149+0555 & \\
\hline 015228 & 2208 & & $1.2 \pm 0.09$ & $1.3 \pm 0.2$ & $1.3 \pm 0.1$ & $1.4 \pm 0.2$ & $1.7 \pm 0.5$ & $0.2 \pm 0.3$ & GB6 J0152+2206 & \\
\hline 020449 & 1513 & 092 & $1.3 \pm 0.06$ & $1.3 \pm 0.1$ & $1.1 \pm 0.1$ & $1.6 \pm 0.3$ & $\ldots$ & $0.0 \pm 0.3$ & GB6 J0204+1514 & $\mathrm{v}$ \\
\hline 020501 & 3213 & 085 & $1.6 \pm 0.07$ & $1.5 \pm 0.1$ & $1.2 \pm 0.1$ & $0.7 \pm 0.2$ & $\ldots$ & $-0.5 \pm 0.3$ & GB6 J0205+3212 & \\
\hline 020503 & -1704 & & $0.7 \pm 0.1$ & $\ldots$ & $0.9 \pm 0.2$ & $0.8 \pm 0.1$ & $0.6 \pm 0.3$ & $0.0 \pm 0.5$ & PMN J0204-1701 & \\
\hline 021051 & -5100 & 158 & $2.7 \pm 0.05$ & $2.7 \pm 0.08$ & $2.8 \pm 0.09$ & $2.7 \pm 0.2$ & $2.1 \pm 0.4$ & $0.0 \pm 0.1$ & PMN J0210-5101 & \\
\hline 021827 & 0138 & 096 & $1.3 \pm 0.05$ & $1.2 \pm 0.08$ & $0.8 \pm 0.1$ & $\ldots$ & $0.7 \pm 0.3$ & $-0.5 \pm 0.3$ & $\ldots$ & $\mathrm{v}$ \\
\hline 022057 & 3558 & & $1.2 \pm 0.06$ & $1.2 \pm 0.09$ & $0.9 \pm 0.1$ & $1.1 \pm 0.2$ & $1.3 \pm 0.3$ & $-0.1 \pm 0.2$ & GB6 J0221+3556 & \\
\hline 022245 & -3440 & 137 & $1.0 \pm 0.03$ & $1.0 \pm 0.05$ & & $0.7 \pm 0.1$ & $\ldots$ & $-0.2 \pm 0.3$ & PMN J0222-3441 & \\
\hline 022310 & 4303 & 084 & $1.8 \pm 0.06$ & $1.4 \pm 0.1$ & $1.4 \pm 0.1$ & $1.3 \pm 0.3$ & $1.2 \pm 0.2$ & $-0.4 \pm 0.2$ & GB6 J0223+4259 & a \\
\hline 023137 & 1320 & & $1.3 \pm 0.07$ & $1.2 \pm 0.08$ & $1.2 \pm 0.1$ & $0.9 \pm 0.2$ & $\ldots$ & $-0.2 \pm 0.3$ & GB6 J0231+1323 & \\
\hline 023139 & -4742 & & $0.7 \pm 0.05$ & $0.9 \pm 0.09$ & $0.9 \pm 0.07$ & $1.2 \pm 0.1$ & $0.7 \pm 0.2$ & $0.3 \pm 0.2$ & PMN J0231-4746 & \\
\hline 023758 & 2848 & 093 & $3.8 \pm 0.06$ & $3.4 \pm 0.1$ & $3.5 \pm 0.1$ & $3.2 \pm 0.3$ & $2.1 \pm 0.4$ & $-0.2 \pm 0.1$ & GB6 J0237+2848 & $\mathrm{v}$ \\
\hline 023848 & 1637 & & $1.5 \pm 0.08$ & $1.6 \pm 0.1$ & $1.6 \pm 0.1$ & $1.6 \pm 0.3$ & $\ldots$ & $0.1 \pm 0.3$ & GB6 J0238+1637 & $\mathrm{v}$ \\
\hline 024118 & -0821 & & $1.0 \pm 0.06$ & $0.7 \pm 0.09$ & $0.7 \pm 0.1$ & & $\ldots$ & $-0.8 \pm 0.5$ & PMN J0241-0815 & $\mathrm{v}$ \\
\hline 024518 & -4456 & & $0.5 \pm 0.05$ & $0.7 \pm 0.1$ & $0.6 \pm 0.09$ & $0.7 \pm 0.2$ & $\ldots$ & $0.5 \pm 0.5$ & PMN J0245-4459 & \\
\hline 025333 & -5442 & 155 & $2.4 \pm 0.04$ & $2.7 \pm 0.07$ & $2.5 \pm 0.08$ & $2.2 \pm 0.1$ & $1.8 \pm 0.3$ & $0.0 \pm 0.1$ & PMN J0253-5441 & $\mathrm{v}$ \\
\hline 025931 & -0015 & & $1.1 \pm 0.06$ & $1.4 \pm 0.08$ & $1.2 \pm 0.08$ & $0.8 \pm 0.1$ & $\ldots$ & $0.0 \pm 0.3$ & PMN J0259-0020 & \\
\hline 030333 & -6212 & 162 & $1.4 \pm 0.06$ & $1.3 \pm 0.1$ & $1.4 \pm 0.08$ & $1.3 \pm 0.1$ & $1.0 \pm 0.2$ & $-0.1 \pm 0.2$ & PMN J0303-6211 & \\
\hline 030826 & 0405 & 102 & $1.3 \pm 0.07$ & $1.3 \pm 0.1$ & $1.2 \pm 0.1$ & $0.9 \pm 0.3$ & $\ldots$ & $-0.1 \pm 0.4$ & GB6 J0308+0406 & \\
\hline 030916 & 1028 & & $1.1 \pm 0.07$ & $1.3 \pm 0.1$ & $1.3 \pm 0.1$ & $1.5 \pm 0.2$ & $1.5 \pm 0.5$ & $0.3 \pm 0.3$ & GB6 J0309+1029 & \\
\hline 030950 & -6102 & 160 & $1.1 \pm 0.05$ & $1.2 \pm 0.08$ & $0.9 \pm 0.07$ & $0.9 \pm 0.2$ & $0.7 \pm 0.3$ & $-0.2 \pm 0.3$ & PMN J0309-6058 & \\
\hline 031221 & -7645 & 174 & $1.0 \pm 0.05$ & $1.2 \pm 0.08$ & $1.1 \pm 0.07$ & $0.9 \pm 0.1$ & $0.8 \pm 0.3$ & $-0.0 \pm 0.2$ & PMN J0311-7651 & \\
\hline 031250 & 0131 & & $0.9 \pm 0.06$ & $0.8 \pm 0.2$ & $0.9 \pm 0.1$ & $0.8 \pm 0.2$ & & $0.0 \pm 0.4$ & GB6 J0312+0132 & \\
\hline 031945 & 4131 & 094 & $11.3 \pm 0.06$ & $8.9 \pm 0.09$ & $7.5 \pm 0.1$ & $5.6 \pm 0.2$ & $3.9 \pm 0.4$ & $-0.7 \pm 0.04$ & GB6 J0319+4130 & $\mathrm{v}$ \\
\hline 032225 & -3711 & 138 & $18.5 \pm 3.1$ & $12.6 \pm 2.0$ & $10.6 \pm 1.9$ & $8.4 \pm 2.5$ & $\ldots$ & $-0.8 \pm 0.2$ & 1Jy 0320-37 & $\mathrm{b}$ \\
\hline 032514 & 2225 & & $0.8 \pm 0.08$ & $0.9 \pm 0.1$ & $1.1 \pm 0.2$ & $0.5 \pm 0.2$ & $\ldots$ & $0.1 \pm 0.5$ & GB6 J0325+2223 & \\
\hline 032945 & -2354 & 123 & $1.2 \pm 0.05$ & $1.3 \pm 0.07$ & $1.2 \pm 0.1$ & $1.0 \pm 0.1$ & $0.9 \pm 0.2$ & $-0.0 \pm 0.2$ & PMN J0329-2357 & \\
\hline 033420 & -4007 & 146 & $1.4 \pm 0.05$ & $1.5 \pm 0.07$ & $1.4 \pm 0.07$ & $1.4 \pm 0.1$ & $1.5 \pm 0.4$ & $0.0 \pm 0.2$ & PMN J0334-4008 & $\mathrm{v}$ \\
\hline 033649 & -1257 & & $1.0 \pm 0.05$ & $0.9 \pm 0.07$ & $1.1 \pm 0.1$ & $0.9 \pm 0.1$ & $0.7 \pm 0.3$ & $-0.1 \pm 0.3$ & PMN J0336-1302 & \\
\hline 033924 & -0143 & 106 & $2.4 \pm 0.07$ & $2.3 \pm 0.1$ & $2.2 \pm 0.1$ & $1.7 \pm 0.2$ & $2.1 \pm 0.3$ & $-0.2 \pm 0.1$ & PMN J0339-0146 & $\mathrm{v}$ \\
\hline 034029 & -2119 & & $1.1 \pm 0.05$ & $1.1 \pm 0.07$ & $1.1 \pm 0.09$ & $1.2 \pm 0.1$ & $1.2 \pm 0.2$ & $0.1 \pm 0.2$ & PMN J0340-2119 & \\
\hline 034851 & -2747 & 129 & $1.2 \pm 0.03$ & $1.0 \pm 0.06$ & $0.9 \pm 0.07$ & $1.5 \pm 0.2$ & $\ldots$ & $-0.2 \pm 0.2$ & PMN J0348-2749 & \\
\hline 035847 & 1029 & & $1.2 \pm 0.1$ & $1.1 \pm 0.2$ & & & $\ldots$ & $-0.1 \pm 1$ & GB6 J0358+1026 & \\
\hline 040357 & -3604 & 136 & $3.4 \pm 0.05$ & $3.8 \pm 0.08$ & $3.6 \pm 0.08$ & $3.4 \pm 0.1$ & $3.0 \pm 0.3$ & $0.0 \pm 0.07$ & PMN J0403-3605 & $\mathrm{v}$ \\
\hline 040536 & -1304 & 114 & $2.0 \pm 0.06$ & $1.8 \pm 0.09$ & $1.7 \pm 0.1$ & $1.5 \pm 0.2$ & $\ldots$ & $-0.3 \pm 0.2$ & PMN J0405-1308 & $\mathrm{v}$ \\
\hline
\end{tabular}


Table 1

(Continued)

\begin{tabular}{|c|c|c|c|c|c|c|c|c|c|c|}
\hline R.A. [hms] & Decl. [dm] & ID & $\mathrm{K}[\mathrm{Jy}]$ & $\mathrm{Ka}[\mathrm{Jy}]$ & Q [Jy] & $\mathrm{V}[\mathrm{Jy}]$ & $\mathrm{W}[\mathrm{Jy}]$ & $\alpha$ & $5 \mathrm{GHz}$ ID & \\
\hline 040702 & -3825 & 141 & $1.1 \pm 0.06$ & $1.0 \pm 0.1$ & $0.9 \pm 0.08$ & $0.8 \pm 0.1$ & $\ldots$ & $-0.4 \pm 0.3$ & PMN J0406-3826 & \\
\hline 040850 & -7506 & & $0.8 \pm 0.04$ & $0.5 \pm 0.06$ & $0.3 \pm 0.08$ & $\ldots$ & $\ldots$ & $-1.4 \pm 0.6$ & PMN J0408-7507 & \\
\hline 041123 & 7654 & 082 & $1.0 \pm 0.05$ & $0.7 \pm 0.1$ & $0.7 \pm 0.1$ & $0.8 \pm 0.2$ & $0.8 \pm 0.2$ & $-0.3 \pm 0.3$ & 1Jy $0403+76$ & \\
\hline 041632 & -2051 & & $1.1 \pm 0.05$ & $1.1 \pm 0.08$ & $1.0 \pm 0.08$ & $0.8 \pm 0.2$ & & $-0.1 \pm 0.3$ & PMN J0416-2056 & \\
\hline 042316 & -0120 & 110 & $8.2 \pm 0.06$ & $8.3 \pm 0.1$ & $7.9 \pm 0.1$ & $7.1 \pm 0.2$ & $4.9 \pm 0.4$ & $-0.1 \pm 0.05$ & PMN J0423-0120 & $\mathrm{v}$ \\
\hline 042350 & 0218 & & $1.2 \pm 0.05$ & $1.0 \pm 0.08$ & $0.7 \pm 0.09$ & $\ldots$ & $\ldots$ & $-0.8 \pm 0.4$ & GB6 J0424+0226 & \\
\hline 042453 & 0035 & 109 & $1.5 \pm 0.08$ & $1.6 \pm 0.1$ & $1.8 \pm 0.1$ & $1.3 \pm 0.2$ & $\ldots$ & $0.2 \pm 0.3$ & GB6 J0424+0036 & $\mathrm{v}$ \\
\hline 042456 & -3757 & 140 & $1.5 \pm 0.05$ & $1.2 \pm 0.1$ & $1.2 \pm 0.1$ & $1.5 \pm 0.2$ & $\ldots$ & $-0.1 \pm 0.2$ & PMN J0424-3756 & $\mathrm{v}$ \\
\hline 042827 & -3757 & & $1.5 \pm 0.05$ & $1.4 \pm 0.08$ & $1.3 \pm 0.07$ & $1.3 \pm 0.2$ & $1.2 \pm 0.4$ & $-0.2 \pm 0.2$ & PMN J0428-3756 & a \\
\hline 043313 & 0521 & 108 & $2.4 \pm 0.06$ & $2.4 \pm 0.1$ & $2.3 \pm 0.1$ & $2.5 \pm 0.3$ & $2.4 \pm 0.4$ & $-0.0 \pm 0.2$ & GB6 J0433+0521 & $\mathrm{v}$ \\
\hline 044016 & -4332 & 147 & $2.5 \pm 0.06$ & $2.3 \pm 0.09$ & $2.2 \pm 0.08$ & $1.7 \pm 0.2$ & $0.9 \pm 0.2$ & $-0.3 \pm 0.1$ & PMN J0440-4332 & $\mathrm{v}$ \\
\hline 044245 & -0017 & & $0.9 \pm 0.06$ & $0.8 \pm 0.1$ & $1.2 \pm 0.2$ & $0.8 \pm 0.2$ & $0.8 \pm 0.3$ & $0.0 \pm 0.4$ & PMN J0442-0017 & \\
\hline 044918 & -8100 & 175 & $1.7 \pm 0.05$ & $1.8 \pm 0.08$ & $1.6 \pm 0.09$ & $1.6 \pm 0.1$ & $1.4 \pm 0.2$ & $-0.0 \pm 0.1$ & PMN J0450-8100 & $\mathrm{v}$ \\
\hline 045319 & -2806 & 131 & $1.5 \pm 0.06$ & $1.5 \pm 0.09$ & $1.4 \pm 0.08$ & $1.2 \pm 0.2$ & $1.5 \pm 0.4$ & $-0.1 \pm 0.2$ & PMN J0453-2807 & \\
\hline 045555 & -4617 & 151 & $3.9 \pm 0.05$ & $4.0 \pm 0.09$ & $4.0 \pm 0.09$ & $3.5 \pm 0.2$ & $2.7 \pm 0.4$ & $-0.0 \pm 0.08$ & PMN J0455-4616 & $\mathrm{V}$ \\
\hline 045659 & -2322 & 128 & $2.4 \pm 0.04$ & $2.5 \pm 0.07$ & $2.4 \pm 0.1$ & $1.9 \pm 0.2$ & $1.9 \pm 0.5$ & $-0.1 \pm 0.1$ & PMN J0457-2324 & $\mathrm{v}$ \\
\hline 050118 & -0159 & & $1.1 \pm 0.07$ & $1.2 \pm 0.1$ & $1.1 \pm 0.1$ & $1.0 \pm 0.3$ & & $-0.0 \pm 0.4$ & PMN J0501-0159 & \\
\hline 050655 & -6108 & 154 & $2.1 \pm 0.04$ & $1.8 \pm 0.06$ & $1.6 \pm 0.07$ & $1.1 \pm 0.1$ & $0.8 \pm 0.2$ & $-0.5 \pm 0.1$ & PMN J0506-6109 & $a, v$ \\
\hline 051356 & -2155 & 127 & $1.1 \pm 0.04$ & $1.1 \pm 0.06$ & $0.9 \pm 0.09$ & & $1.0 \pm 0.3$ & $-0.1 \pm 0.3$ & PMN J0513-2159 & $\mathrm{v}$ \\
\hline 051520 & -4558 & & $\ldots$ & $\ldots$ & $0.9 \pm 0.1$ & $1.1 \pm 0.2$ & $\ldots$ & $0.4 \pm 1$ & PMN J0515-4556 & a \\
\hline 051921 & -0539 & 116 & $2.4 \pm 0.06$ & $1.8 \pm 0.07$ & $1.2 \pm 0.09$ & $0.8 \pm 0.1$ & $\ldots$ & $-1.0 \pm 0.2$ & PMN J0520-0537 & $\mathrm{c}$ \\
\hline 051942 & -4546 & 150 & $6.8 \pm 0.05$ & $5.3 \pm 0.08$ & $4.4 \pm 0.1$ & $3.3 \pm 0.2$ & $2.2 \pm 0.3$ & $-0.7 \pm 0.06$ & PMN J0519-4546 & a \\
\hline 052302 & -3627 & 139 & $4.2 \pm 0.05$ & $3.9 \pm 0.08$ & $3.8 \pm 0.1$ & $3.5 \pm 0.2$ & $2.6 \pm 0.2$ & $-0.2 \pm 0.07$ & PMN J0522-3628 & $\mathrm{v}$ \\
\hline 052505 & -2337 & & $0.7 \pm 0.04$ & $0.9 \pm 0.06$ & $0.7 \pm 0.07$ & $0.8 \pm 0.2$ & $\ldots$ & $0.1 \pm 0.3$ & PMN J0525-2338 & a \\
\hline 052531 & -4826 & & $0.9 \pm 0.04$ & $1.3 \pm 0.07$ & $1.3 \pm 0.09$ & $1.1 \pm 0.1$ & $0.8 \pm 0.2$ & $0.3 \pm 0.2$ & PMN J0526-4830 & $a, v$ \\
\hline 052734 & -1241 & 122 & $1.4 \pm 0.05$ & $1.6 \pm 0.09$ & $1.4 \pm 0.1$ & $1.2 \pm 0.1$ & $1.1 \pm 0.3$ & $-0.1 \pm 0.2$ & PMN J0527-1241 & \\
\hline 053423 & -6107 & & $0.5 \pm 0.03$ & $0.5 \pm 0.05$ & $0.6 \pm 0.05$ & $0.6 \pm 0.09$ & & $0.0 \pm 0.3$ & PMN J0534-6106 & \\
\hline 053852 & -4405 & 148 & $5.6 \pm 0.05$ & $5.9 \pm 0.08$ & $6.0 \pm 0.09$ & $5.4 \pm 0.2$ & $4.6 \pm 0.3$ & $0.0 \pm 0.05$ & PMN J0538-4405 & $\mathrm{v}$ \\
\hline 053948 & -2844 & & $0.6 \pm 0.09$ & $0.6 \pm 0.08$ & $0.6 \pm 0.1$ & $0.7 \pm 0.1$ & $\ldots$ & $0.2 \pm 0.5$ & PMN J0539-2839 & $\mathrm{v}$ \\
\hline 054044 & -5415 & 152 & $1.4 \pm 0.05$ & $1.4 \pm 0.07$ & $1.4 \pm 0.09$ & $1.1 \pm 0.1$ & $\ldots$ & $-0.1 \pm 0.2$ & PMN J0540-5418 & $\mathrm{v}$ \\
\hline 054228 & 4951 & 095 & $1.7 \pm 0.07$ & $1.3 \pm 0.1$ & $1.3 \pm 0.1$ & $0.8 \pm 0.1$ & $\ldots$ & $-0.7 \pm 0.3$ & GB6 J0542+4951 & \\
\hline 055039 & -5731 & 153 & $1.2 \pm 0.04$ & $1.0 \pm 0.05$ & $1.0 \pm 0.08$ & $0.9 \pm 0.1$ & $\ldots$ & $-0.3 \pm 0.2$ & PMN J0550-5732 & \\
\hline 055559 & 3942 & 100 & $3.0 \pm 0.06$ & $2.4 \pm 0.09$ & $1.7 \pm 0.1$ & & $\ldots$ & $-0.8 \pm 0.2$ & GB6 J0555+3948 & \\
\hline 055953 & -4528 & & $0.7 \pm 0.05$ & $1.0 \pm 0.07$ & $0.9 \pm 0.06$ & $0.7 \pm 0.1$ & $\ldots$ & $0.4 \pm 0.4$ & PMN J0559-4529 & \\
\hline 060700 & 6723 & 091 & $1.2 \pm 0.04$ & $0.9 \pm 0.05$ & $0.7 \pm 0.08$ & $0.6 \pm 0.2$ & $\ldots$ & $-0.7 \pm 0.3$ & GB6 J0607+6720 & a \\
\hline 060847 & -2220 & & $1.1 \pm 0.04$ & $1.1 \pm 0.06$ & $0.9 \pm 0.08$ & $0.6 \pm 0.1$ & $0.5 \pm 0.2$ & $-0.3 \pm 0.3$ & PMN J0608-2220 & \\
\hline 060937 & -1541 & 126 & $3.7 \pm 0.05$ & $3.3 \pm 0.09$ & $3.1 \pm 0.1$ & $2.2 \pm 0.2$ & $1.9 \pm 0.5$ & $-0.3 \pm 0.1$ & PMN J0609-1542 & $\mathrm{V}$ \\
\hline 062102 & -2516 & & $0.6 \pm 0.06$ & $0.5 \pm 0.1$ & $0.3 \pm 0.1$ & $\ldots$ & 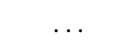 & $-1.0 \pm 1$ & PMN J0620-2515 & \\
\hline 062303 & -6436 & & $0.9 \pm 0.03$ & $0.8 \pm 0.05$ & $0.8 \pm 0.04$ & $0.9 \pm 0.06$ & $0.9 \pm 0.1$ & $-0.1 \pm 0.1$ & PMN J0623-6436 & $\mathrm{v}$ \\
\hline 062634 & -3523 & & $0.7 \pm 0.06$ & $0.5 \pm 0.1$ & & $\ldots$ & $\ldots$ & $-0.9 \pm 2$ & PMN J0627-3529 & \\
\hline 062928 & -1958 & 130 & $1.5 \pm 0.04$ & $1.4 \pm 0.07$ & $1.4 \pm 0.1$ & $1.2 \pm 0.2$ & $1.1 \pm 0.3$ & $-0.2 \pm 0.2$ & PMN J0629-1959 & $\mathrm{v}$ \\
\hline 063221 & -6928 & & $0.4 \pm 0.03$ & $0.5 \pm 0.04$ & $0.4 \pm 0.04$ & $0.7 \pm 0.1$ & $0.6 \pm 0.2$ & $0.5 \pm 0.3$ & & \\
\hline 063351 & -2218 & 135 & $0.5 \pm 0.06$ & $0.6 \pm 0.07$ & $0.7 \pm 0.1$ & $0.8 \pm 0.2$ & $0.9 \pm 0.2$ & $0.5 \pm 0.4$ & PMN J0633-2223 & $\mathrm{v}$ \\
\hline 063438 & -2337 & & $0.6 \pm 0.05$ & $0.7 \pm 0.08$ & $0.6 \pm 0.08$ & $0.6 \pm 0.2$ & $\ldots$ & $0.1 \pm 0.5$ & PMN J0634-2335 & \\
\hline 063551 & -7517 & 167 & $4.3 \pm 0.04$ & $3.9 \pm 0.06$ & $3.6 \pm 0.07$ & $2.6 \pm 0.1$ & $2.5 \pm 0.4$ & $-0.3 \pm 0.06$ & PMN J0635-7516 & $\mathrm{v}$ \\
\hline 063631 & -2031 & 134 & $1.1 \pm 0.04$ & $1.1 \pm 0.06$ & $1.0 \pm 0.08$ & $0.7 \pm 0.1$ & $\ldots$ & $-0.3 \pm 0.3$ & PMN J0636-2041 & a \\
\hline 063939 & 7327 & 087 & $0.8 \pm 0.05$ & $0.4 \pm 0.1$ & $0.8 \pm 0.1$ & $0.8 \pm 0.1$ & $1.0 \pm 0.2$ & $0.1 \pm 0.3$ & GB6 J0639+7324 & \\
\hline 064630 & 4449 & 099 & $2.9 \pm 0.06$ & $2.4 \pm 0.1$ & $2.1 \pm 0.1$ & $1.6 \pm 0.2$ & $1.3 \pm 0.3$ & $-0.5 \pm 0.2$ & GB6 J0646+4451 & $\mathrm{v}$ \\
\hline 072005 & -6222 & & $0.6 \pm 0.04$ & $0.7 \pm 0.06$ & $0.8 \pm 0.05$ & $0.5 \pm 0.09$ & $0.7 \pm 0.3$ & $0.2 \pm 0.3$ & PMN J0719-6218 & \\
\hline 072036 & 0403 & & $0.9 \pm 0.05$ & $0.7 \pm 0.08$ & $0.6 \pm 0.1$ & $\ldots$ & 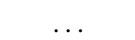 & $-0.6 \pm 0.5$ & GB6 J0720+0404 & \\
\hline 072154 & 7122 & & $1.6 \pm 0.04$ & $1.8 \pm 0.07$ & $1.9 \pm 0.08$ & $1.8 \pm 0.2$ & $1.5 \pm 0.2$ & $0.2 \pm 0.1$ & GB6 J0721+7120 & $\mathrm{v}$ \\
\hline 072552 & -0050 & & $0.9 \pm 0.1$ & $1.2 \pm 0.1$ & $1.1 \pm 0.1$ & $1.0 \pm 0.3$ & $1.1 \pm 0.2$ & $0.1 \pm 0.4$ & PMN J0725-0054 & \\
\hline 072709 & 6749 & & $0.6 \pm 0.05$ & $0.5 \pm 0.08$ & $0.6 \pm 0.1$ & $0.8 \pm 0.3$ & $2.2-0.0$ & $-0.0 \pm 0.5$ & GB6 J0728+6748 & \\
\hline 073813 & 1743 & 113 & $1.3 \pm 0.06$ & $1.3 \pm 0.1$ & $1.0 \pm 0.1$ & $1.3 \pm 0.3$ & $\ldots$ & $-0.2 \pm 0.3$ & GB6 J0738+1742 & $\mathrm{v}$ \\
\hline 073915 & 0136 & 124 & $1.7 \pm 0.06$ & $1.9 \pm 0.1$ & $2.1 \pm 0.1$ & $2.3 \pm 0.2$ & $2.8 \pm 1$ & $0.3 \pm 0.2$ & GB6 J0739+0136 & $\mathrm{v}$ \\
\hline 074118 & 3111 & 107 & $1.2 \pm 0.06$ & $1.1 \pm 0.1$ & $0.8 \pm 0.1$ & $1.0 \pm 0.3$ & $\ldots$ & $-0.3 \pm 0.4$ & GB6 J0741+3112 & \\
\hline 074349 & -6727 & 161 & $1.2 \pm 0.04$ & $0.9 \pm 0.07$ & $0.7 \pm 0.08$ & $0.7 \pm 0.2$ & $1.0 \pm 0.2$ & $-0.5 \pm 0.2$ & PMN J0743-6726 & \\
\hline 074524 & 1016 & 118 & $1.1 \pm 0.06$ & $0.8 \pm 0.1$ & $0.7 \pm 0.1$ & $1.0 \pm 0.4$ & $\ldots$ & $-0.5 \pm 0.5$ & GB6 J0745+1011 & \\
\hline 074604 & -0045 & & $1.1 \pm 0.07$ & $1.0 \pm 0.1$ & $0.8 \pm 0.1$ & $0.7 \pm 0.1$ & $\ldots$ & $-0.5 \pm 0.4$ & PMN J0745-0044 & \\
\hline 075052 & 1230 & 117 & $2.7 \pm 0.06$ & $2.6 \pm 0.1$ & $2.6 \pm 0.1$ & $2.1 \pm 0.2$ & $1.8 \pm 0.3$ & $-0.2 \pm 0.1$ & GB6 J0750+1231 & $\mathrm{v}$ \\
\hline 075332 & 5354 & & $1.0 \pm 0.06$ & $1.0 \pm 0.08$ & $0.9 \pm 0.09$ & $0.9 \pm 0.2$ & $\ldots$ & $-0.2 \pm 0.3$ & GB6 J0753+5353 & a \\
\hline 075703 & 0957 & 120 & $1.3 \pm 0.08$ & $1.3 \pm 0.1$ & $1.5 \pm 0.1$ & $1.4 \pm 0.3$ & $\ldots$ & $0.2 \pm 0.3$ & GB6 J0757+0956 & $\mathrm{v}$ \\
\hline 080543 & 6133 & & $0.7 \pm 0.05$ & $0.5 \pm 0.06$ & $0.7 \pm 0.08$ & $1.0 \pm 0.3$ & $\ldots$ & $0.0 \pm 0.4$ & $\ldots$ & \\
\hline
\end{tabular}


Table 1

(Continued)

\begin{tabular}{|c|c|c|c|c|c|c|c|c|c|c|}
\hline R.A. [hms] & Decl. [dm] & ID & $\mathrm{K}[\mathrm{Jy}]$ & $\mathrm{Ka}[\mathrm{Jy}]$ & Q [Jy] & $\mathrm{V}[\mathrm{Jy}]$ & W [Jy] & $\alpha$ & $5 \mathrm{GHz}$ ID & \\
\hline 080822 & -0750 & 133 & $1.3 \pm 0.05$ & $1.3 \pm 0.08$ & $1.2 \pm 0.1$ & $1.3 \pm 0.2$ & $0.9 \pm 0.2$ & $-0.1 \pm 0.2$ & PMN J0808-0751 & $\mathrm{v}$ \\
\hline 081326 & 4817 & & $1.0 \pm 0.06$ & $1.0 \pm 0.09$ & $0.7 \pm 0.1$ & $0.7 \pm 0.2$ & $\ldots$ & $-0.4 \pm 0.4$ & GB6 J0813+4813 & \\
\hline 081619 & -2425 & 145 & $0.8 \pm 0.05$ & $1.0 \pm 0.06$ & $1.1 \pm 0.08$ & $0.8 \pm 0.2$ & $\ldots$ & $0.3 \pm 0.3$ & PMN J0816-2421 & $\mathrm{v}$ \\
\hline 082326 & 2225 & & $1.1 \pm 0.06$ & $1.2 \pm 0.09$ & $1.2 \pm 0.1$ & $0.6 \pm 0.2$ & & $0.0 \pm 0.4$ & GB6 J0823+2223 & $\dagger$ \\
\hline 082454 & 3914 & & $1.2 \pm 0.07$ & $1.0 \pm 0.1$ & $1.1 \pm 0.1$ & $1.1 \pm 0.2$ & $1.4 \pm 0.7$ & $-0.1 \pm 0.3$ & GB6 J0824+3916 & a \\
\hline 082550 & 0311 & 125 & $1.6 \pm 0.06$ & $1.9 \pm 0.09$ & $1.9 \pm 0.1$ & $1.6 \pm 0.2$ & & $0.1 \pm 0.2$ & GB6 J0825+0309 & $\mathrm{v}$ \\
\hline 083100 & 2411 & 112 & $1.3 \pm 0.08$ & $1.3 \pm 0.1$ & $1.5 \pm 0.2$ & $1.5 \pm 0.2$ & $1.3 \pm 0.3$ & $0.2 \pm 0.3$ & GB6 J0830+2410 & $\mathrm{v}$ \\
\hline 083451 & 5533 & & $0.9 \pm 0.06$ & $0.7 \pm 0.08$ & $0.5 \pm 0.1$ & $1.1 \pm 0.4$ & $0.7 \pm 0.3$ & $-0.4 \pm 0.5$ & GB6 J0834+5534 & \\
\hline 083647 & -2015 & 144 & $2.8 \pm 0.05$ & $2.4 \pm 0.09$ & $2.3 \pm 0.09$ & $1.8 \pm 0.2$ & $0.8 \pm 0.3$ & $-0.4 \pm 0.1$ & PMN J0836-2017 & $\mathrm{v}$ \\
\hline 083811 & 5822 & & $1.1 \pm 0.04$ & $1.1 \pm 0.07$ & $0.9 \pm 0.09$ & $1.0 \pm 0.2$ & $\ldots$ & $-0.2 \pm 0.3$ & GB6 J0837+5825 & a \\
\hline 084042 & 1312 & 121 & $1.9 \pm 0.07$ & $2.0 \pm 0.1$ & $1.8 \pm 0.1$ & $1.0 \pm 0.2$ & $\ldots$ & $-0.1 \pm 0.2$ & GB6 J0840+1312 & $\mathrm{v}$ \\
\hline 084128 & 7053 & 089 & $1.7 \pm 0.04$ & $1.7 \pm 0.07$ & $1.8 \pm 0.09$ & $1.5 \pm 0.2$ & $0.5 \pm 0.2$ & $-0.1 \pm 0.2$ & GB6 J0841+7053 & $\mathrm{v}$ \\
\hline 084745 & -0704 & & $0.9 \pm 0.06$ & $1.0 \pm 0.09$ & $1.0 \pm 0.1$ & $1.2 \pm 0.3$ & 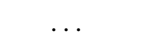 & $0.2 \pm 0.4$ & PMN J0847-0703 & \\
\hline 085447 & 2006 & 115 & $3.8 \pm 0.07$ & $4.4 \pm 0.1$ & $4.1 \pm 0.1$ & $4.2 \pm 0.2$ & $3.5 \pm 0.5$ & $0.1 \pm 0.1$ & GB6 J0854+2006 & V \\
\hline 090217 & -1413 & & $1.2 \pm 0.06$ & $1.3 \pm 0.08$ & $1.2 \pm 0.09$ & $1.3 \pm 0.1$ & $\ldots$ & $0.1 \pm 0.2$ & PMN J0902-1415 & \\
\hline 090758 & -2020 & & $1.1 \pm 0.05$ & $1.0 \pm 0.09$ & $0.7 \pm 0.1$ & $1.0 \pm 0.1$ & $\ldots$ & $-0.3 \pm 0.3$ & PMN J0907-2026 & \\
\hline 090916 & 0119 & 132 & $2.1 \pm 0.06$ & $2.0 \pm 0.1$ & $1.9 \pm 0.2$ & $2.0 \pm 0.2$ & $\ldots$ & $-0.1 \pm 0.2$ & GB6 J0909+0121 & $\mathrm{v}$ \\
\hline 090948 & 4253 & & $1.0 \pm 0.07$ & $1.1 \pm 0.1$ & $1.2 \pm 0.1$ & $0.9 \pm 0.2$ & $\ldots$ & $0.2 \pm 0.4$ & GB6 J0909+4253 & \\
\hline 091441 & 0248 & & $1.4 \pm 0.06$ & $1.6 \pm 0.1$ & $1.4 \pm 0.1$ & $0.8 \pm 0.4$ & $1.4 \pm 0.3$ & $0.0 \pm 0.2$ & GB6 J0914+0245 & \\
\hline 091810 & -1203 & 143 & $2.0 \pm 0.07$ & $1.0 \pm 0.1$ & $0.9 \pm 0.2$ & $0.9 \pm 0.3$ & $\ldots$ & $-1.3 \pm 0.5$ & PMN J0918-1205 & \\
\hline 092040 & 4441 & & $1.3 \pm 0.07$ & $1.4 \pm 0.1$ & $1.4 \pm 0.1$ & $1.4 \pm 0.2$ & $0.6 \pm 0.3$ & $0.0 \pm 0.3$ & GB6 J0920+4441 & $\mathrm{v}$ \\
\hline 092105 & 6215 & & $0.9 \pm 0.05$ & $0.8 \pm 0.07$ & $0.9 \pm 0.1$ & $\ldots$ & $\ldots$ & $-0.1 \pm 0.5$ & GB6 J0921+6215 & \\
\hline 092139 & -2619 & & $1.5 \pm 0.05$ & $1.4 \pm 0.08$ & $1.3 \pm 0.1$ & $1.2 \pm 0.2$ & $0.8 \pm 0.3$ & $-0.2 \pm 0.2$ & PMN J0921-2618 & $\mathrm{v}$ \\
\hline 092705 & 3901 & 105 & $6.7 \pm 0.07$ & $5.8 \pm 0.1$ & $5.5 \pm 0.1$ & $4.4 \pm 0.2$ & $2.8 \pm 0.3$ & $-0.4 \pm 0.07$ & GB6 J0927+3902 & $\mathrm{v}$ \\
\hline 094853 & 4038 & 104 & $1.3 \pm 0.06$ & $1.5 \pm 0.1$ & $1.3 \pm 0.1$ & $0.9 \pm 0.2$ & $1.0 \pm 0.3$ & $-0.1 \pm 0.3$ & GB6 J0948+4039 & $\mathrm{v}$ \\
\hline 095547 & 6935 & 088 & $1.3 \pm 0.05$ & $1.2 \pm 0.07$ & $1.0 \pm 0.07$ & $0.9 \pm 0.1$ & $1.3 \pm 0.4$ & $-0.3 \pm 0.2$ & GB6 J0955+6940 & \\
\hline 095724 & 5527 & & $0.9 \pm 0.05$ & $0.9 \pm 0.1$ & $1.0 \pm 0.1$ & $0.8 \pm 0.2$ & $\ldots$ & $-0.0 \pm 0.4$ & GB6 J0957+5522 & $\mathrm{a}$ \\
\hline 095808 & 4722 & 098 & $1.6 \pm 0.06$ & $1.4 \pm 0.09$ & $1.4 \pm 0.09$ & $0.9 \pm 0.1$ & $\ldots$ & $-0.4 \pm 0.2$ & GB6 J0958+4725 & \\
\hline 095925 & 6530 & & $0.9 \pm 0.05$ & $0.9 \pm 0.06$ & $0.7 \pm 0.08$ & $0.8 \pm 0.1$ & $0.8 \pm 0.2$ & $-0.2 \pm 0.3$ & GB6 J0958+6534 & $\mathrm{v}$ \\
\hline 101401 & 2306 & 119 & $1.1 \pm 0.06$ & $0.9 \pm 0.1$ & $0.7 \pm 0.07$ & $0.7 \pm 0.2$ & $\ldots$ & $-0.6 \pm 0.3$ & & \\
\hline 101520 & -4511 & & $1.1 \pm 0.04$ & $0.8 \pm 0.06$ & $0.7 \pm 0.08$ & & $\ldots$ & $-0.8 \pm 0.4$ & PMN J1014-4508 & \\
\hline 101737 & 3551 & & $0.9 \pm 0.05$ & $0.9 \pm 0.08$ & $0.8 \pm 0.1$ & $0.6 \pm 0.2$ & $0.9 \pm 0.3$ & $-0.1 \pm 0.3$ & GB6 J1018+3550 & \\
\hline 101853 & -3132 & & $0.9 \pm 0.05$ & $0.9 \pm 0.07$ & $0.7 \pm 0.09$ & $0.6 \pm 0.2$ & $\ldots$ & $-0.3 \pm 0.4$ & PMN J1018-3123 & \\
\hline 102133 & 4003 & & $0.9 \pm 0.04$ & $0.9 \pm 0.07$ & $0.9 \pm 0.08$ & & $\ldots$ & $-0.1 \pm 0.4$ & GB6 J1022+4004 & \\
\hline 103233 & 4118 & 103 & $0.9 \pm 0.05$ & $0.8 \pm 0.09$ & $0.7 \pm 0.1$ & $0.8 \pm 0.2$ & $0.8 \pm 0.3$ & $-0.2 \pm 0.3$ & GB6 J1033+4115 & $\mathrm{v}$ \\
\hline 103722 & -2934 & & $1.6 \pm 0.06$ & $1.6 \pm 0.09$ & $1.4 \pm 0.1$ & $1.6 \pm 0.3$ & $1.7 \pm 0.3$ & $0.0 \pm 0.2$ & PMN J1037-2934 & V \\
\hline 103838 & 0510 & 142 & $1.4 \pm 0.06$ & $1.5 \pm 0.1$ & $1.1 \pm 0.2$ & $1.1 \pm 0.2$ & $0.9 \pm 0.3$ & $-0.3 \pm 0.3$ & GB6 J1038+0512 & $\mathrm{v}$ \\
\hline 104127 & 0611 & & $1.2 \pm 0.07$ & $1.4 \pm 0.1$ & $1.2 \pm 0.1$ & $1.1 \pm 0.2$ & $\ldots$ & $0.0 \pm 0.3$ & GB6 J1041+0610 & \\
\hline 104138 & -4738 & 163 & $1.1 \pm 0.05$ & $\ldots$ & $0.5 \pm 0.06$ & $\ldots$ & $\ldots$ & $-1.3 \pm 0.5$ & PMN J1041-4740 & \\
\hline 104301 & 2407 & & $0.8 \pm 0.08$ & $0.8 \pm 0.1$ & $0.8 \pm 0.1$ & $\ldots$ & & $-0.0 \pm 0.7$ & GB6 J1043+2408 & \\
\hline 104744 & 7143 & 083 & $1.4 \pm 0.06$ & $1.4 \pm 0.1$ & $1.2 \pm 0.1$ & $1.3 \pm 0.3$ & & $-0.1 \pm 0.3$ & GB6 J1048+7143 & $\mathrm{V}$ \\
\hline 104756 & -1909 & & $1.3 \pm 0.06$ & $1.1 \pm 0.09$ & $1.1 \pm 0.2$ & $1.2 \pm 0.3$ & $\ldots$ & $-0.3 \pm 0.4$ & PMN J1048-1909 & \\
\hline 105329 & 8109 & & $0.9 \pm 0.0$ & $0.9 \pm 0.07$ & & $\ldots$ & $\ldots$ & $-0.1 \pm 0.6$ & ( & \\
\hline 105827 & 0134 & 149 & $4.6 \pm 0.06$ & $4.4 \pm 0.09$ & $4.5 \pm 0.1$ & $4.4 \pm 0.2$ & $3.1 \pm 0.9$ & $-0.1 \pm 0.08$ & GB6 J1058+0133 & $\mathrm{V}$ \\
\hline 105922 & -8003 & 176 & $2.1 \pm 0.05$ & $2.3 \pm 0.07$ & $2.2 \pm 0.08$ & $2.3 \pm 0.2$ & $1.4 \pm 0.3$ & $0.0 \pm 0.1$ & PMN J1058-8003 & $\mathrm{V}$ \\
\hline 110213 & -4403 & & $0.7 \pm 0.04$ & $0.8 \pm 0.05$ & $0.8 \pm 0.1$ & $0.8 \pm 0.1$ & & $0.2 \pm 0.3$ & PMN J1102-4404 & \\
\hline 110711 & -4446 & 166 & $1.5 \pm 0.04$ & $1.5 \pm 0.05$ & $1.2 \pm 0.07$ & $1.4 \pm 0.2$ & $0.9 \pm 0.2$ & $-0.3 \pm 0.2$ & PMN J1107-4449 & $\mathrm{v}$ \\
\hline 111806 & -4633 & & $1.0 \pm 0.04$ & $0.8 \pm 0.07$ & $0.7 \pm 0.07$ & $0.7 \pm 0.3$ & $\ldots$ & $-0.5 \pm 0.3$ & PMN J1118-4634 & \\
\hline 111833 & -1233 & & $1.0 \pm 0.06$ & $0.9 \pm 0.07$ & $0.8 \pm 0.09$ & $0.9 \pm 0.2$ & $\ldots$ & $-0.3 \pm 0.3$ & PMN J1118-1232 & a \\
\hline 111846 & 1240 & & $0.9 \pm 0.06$ & $0.9 \pm 0.1$ & $0.7 \pm 0.1$ & $0.8 \pm 0.2$ & $\ldots$ & $-0.3 \pm 0.5$ & GB6 J1118+1234 & a \\
\hline 112706 & -1858 & 159 & $1.5 \pm 0.06$ & $1.5 \pm 0.1$ & $1.4 \pm 0.1$ & $1.1 \pm 0.2$ & $1.3 \pm 0.3$ & $-0.1 \pm 0.2$ & PMN J1127-1857 & \\
\hline 113012 & -1451 & 157 & $1.8 \pm 0.06$ & $1.7 \pm 0.1$ & $1.9 \pm 0.1$ & $1.3 \pm 0.2$ & $\ldots$ & $-0.1 \pm 0.2$ & PMN J1130-1449 & $\mathrm{v}$ \\
\hline 113045 & 3814 & 101 & $1.2 \pm 0.06$ & $1.0 \pm 0.09$ & $1.1 \pm 0.1$ & $0.8 \pm 0.2$ & $0.9 \pm 0.3$ & $-0.3 \pm 0.3$ & GB6 J1130+3815 & a \\
\hline 113657 & -7416 & & $0.8 \pm 0.04$ & $0.7 \pm 0.07$ & $0.5 \pm 0.09$ & $0.5 \pm 0.2$ & 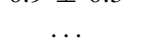 & $-0.5 \pm 0.5$ & PMN J1136-7415 & \\
\hline 114620 & -4842 & & $0.7 \pm 0.04$ & $0.7 \pm 0.06$ & $0.5 \pm 0.08$ & $0.8 \pm 0.1$ & $\cdots$ & $0.0 \pm 0.3$ & PMN J1145-4836 & a \\
\hline 114643 & 4001 & & $0.9 \pm 0.06$ & $1.0 \pm 0.07$ & $1.1 \pm 0.07$ & $0.4 \pm 0.1$ & $\ldots$ & $0.1 \pm 0.3$ & GB6 J1146+3958 & a \\
\hline 114707 & -3811 & 169 & $2.1 \pm 0.05$ & $2.3 \pm 0.09$ & $2.2 \pm 0.09$ & $1.9 \pm 0.2$ & $1.1 \pm 0.2$ & $-0.1 \pm 0.1$ & PMN J1147-3812 & $\mathrm{V}$ \\
\hline 115012 & -7927 & & $1.2 \pm 0.04$ & $0.7 \pm 0.06$ & $0.6 \pm 0.08$ & $0.6 \pm 0.1$ & $\ldots$ & $-1.1 \pm 0.3$ & PMN J1150-7918 & \\
\hline 115053 & -0024 & & $0.8 \pm 0.08$ & $0.7 \pm 0.1$ & $0.7 \pm 0.2$ & $\ldots$ & $\ldots$ & $-0.2 \pm 0.8$ & PMN J1150-0024 & \\
\hline 115315 & 4932 & 090 & $2.1 \pm 0.04$ & $2.2 \pm 0.07$ & $2.2 \pm 0.08$ & $2.0 \pm 0.2$ & $1.2 \pm 0.3$ & $-0.0 \pm 0.1$ & GB6 J1153+4931 & $\mathrm{a}, \mathrm{v}$ \\
\hline 115502 & 8104 & 078 & $1.2 \pm 0.06$ & $1.0 \pm 0.1$ & $0.9 \pm 0.09$ & $1.0 \pm 0.2$ & $1.1 \pm 0.3$ & $-0.2 \pm 0.3$ & 1Jy 1150+81 & $\mathrm{v}$ \\
\hline 115744 & 1638 & & $0.8 \pm 0.05$ & $1.1 \pm 0.09$ & $0.8 \pm 0.09$ & $0.8 \pm 0.2$ & $0.7 \pm 0.3$ & $0.0 \pm 0.3$ & GB6 J1157+1639 & \\
\hline 115935 & 2915 & 111 & $2.0 \pm 0.05$ & $2.2 \pm 0.09$ & $2.2 \pm 0.1$ & $2.0 \pm 0.2$ & $2.0 \pm 0.4$ & $0.0 \pm 0.1$ & GB6 J1159+2914 & $\mathrm{v}$ \\
\hline 120330 & 4808 & & $0.8 \pm 0.04$ & $0.7 \pm 0.06$ & $0.6 \pm 0.08$ & $0.7 \pm 0.3$ & $0.6 \pm 0.2$ & $-0.3 \pm 0.3$ & GB6 J1203+4803 & a \\
\hline 120902 & -2403 & 172 & $1.3 \pm 0.06$ & $1.0 \pm 0.09$ & $0.9 \pm 0.09$ & $\ldots$ & $\ldots$ & $-0.6 \pm 0.4$ & PMN J1209-2406 & \\
\hline
\end{tabular}


Table 1

(Continued)

\begin{tabular}{|c|c|c|c|c|c|c|c|c|c|c|}
\hline R.A. [hms] & Decl. [dm] & ID & $\mathrm{K}[\mathrm{Jy}]$ & $\mathrm{Ka}[\mathrm{Jy}]$ & Q [Jy] & $\mathrm{V}$ [Jy] & $\mathrm{W}[\mathrm{Jy}]$ & $\alpha$ & $5 \mathrm{GHz}$ ID & \\
\hline 121556 & -1729 & 173 & $1.4 \pm 0.06$ & $1.2 \pm 0.1$ & $1.1 \pm 0.1$ & $0.8 \pm 0.2$ & & $-0.4 \pm 0.3$ & PMN J1215-1731 & \\
\hline 121853 & 4830 & & $0.7 \pm 0.03$ & $0.7 \pm 0.05$ & $0.7 \pm 0.08$ & $0.7 \pm 0.1$ & $0.6 \pm 0.2$ & $0.0 \pm 0.3$ & GB6 J1219+4830 & \\
\hline 121921 & 0549 & 164 & $2.7 \pm 0.06$ & $2.2 \pm 0.1$ & $2.0 \pm 0.1$ & $1.4 \pm 0.2$ & $2.0 \pm 0.6$ & $-0.5 \pm 0.2$ & GB6 J1219+0549A & a \\
\hline 122206 & 0414 & & $0.7 \pm 0.07$ & $0.7 \pm 0.1$ & $1.0 \pm 0.2$ & & & $0.4 \pm 0.7$ & GB6 J1222+0413 & \\
\hline 122353 & -8306 & 178 & $0.8 \pm 0.06$ & $0.9 \pm 0.06$ & $0.8 \pm 0.06$ & $0.7 \pm 0.1$ & $0.8 \pm 0.3$ & $-0.1 \pm 0.3$ & PMN J1224-8312 & \\
\hline 122906 & 0203 & 170 & $20.0 \pm 0.06$ & $18.4 \pm 0.1$ & $16.8 \pm 0.1$ & $14.6 \pm 0.2$ & $10.5 \pm 0.4$ & $-0.3 \pm 0.02$ & GB6 J1229+0202 & $\mathrm{V}$ \\
\hline 123051 & 1223 & 165 & $19.7 \pm 0.06$ & $15.5 \pm 0.09$ & $13.3 \pm 0.1$ & $9.7 \pm 0.2$ & $6.2 \pm 0.4$ & $-0.7 \pm 0.02$ & GB6 J1230+1223 & $\mathrm{v}$ \\
\hline 123925 & 0728 & & $1.0 \pm 0.06$ & $1.0 \pm 0.1$ & $0.9 \pm 0.09$ & $1.0 \pm 0.2$ & $\ldots$ & $-0.2 \pm 0.3$ & GB6 J1239+0730 & \\
\hline 124653 & -2547 & 177 & $1.3 \pm 0.06$ & $1.4 \pm 0.1$ & $1.6 \pm 0.1$ & $1.5 \pm 0.2$ & $1.4 \pm 0.5$ & $0.2 \pm 0.2$ & PMN J1246-2547 & $\mathrm{v}$ \\
\hline 124850 & -4600 & & $0.8 \pm 0.08$ & $0.8 \pm 0.1$ & $0.9 \pm 0.09$ & $0.9 \pm 0.2$ & $1.4 \pm 0.3$ & $0.3 \pm 0.3$ & PMN J1248-4559 & $\mathrm{v}$ \\
\hline 125450 & 1142 & & $0.9 \pm 0.06$ & $0.9 \pm 0.08$ & $0.8 \pm 0.09$ & $\ldots$ & $\ldots$ & $-0.2 \pm 0.4$ & GB6 J1254+1141 & \\
\hline 125612 & -0547 & 181 & $17.1 \pm 0.06$ & $17.9 \pm 0.1$ & $18.2 \pm 0.1$ & $17.0 \pm 0.2$ & $13.3 \pm 0.4$ & $0.0 \pm 0.02$ & PMN J1256-0547 & V \\
\hline 125809 & -3158 & 180 & $1.3 \pm 0.05$ & $1.1 \pm 0.07$ & $1.1 \pm 0.1$ & $0.5 \pm 0.2$ & $1.6 \pm 0.5$ & $-0.2 \pm 0.3$ & PMN J1257-3154 & $\mathrm{v}$ \\
\hline 125826 & 3226 & & $0.7 \pm 0.05$ & $0.6 \pm 0.09$ & $0.8 \pm 0.1$ & $0.5 \pm 0.2$ & $0.5 \pm 0.2$ & $-0.1 \pm 0.5$ & GB6 J1257+3229 & a \\
\hline 125854 & -2223 & & $1.0 \pm 0.06$ & $0.8 \pm 0.1$ & $0.8 \pm 0.1$ & $0.7 \pm 0.2$ & $\ldots$ & $-0.3 \pm 0.4$ & PMN J1258-2219 & \\
\hline 125927 & 5141 & & $0.6 \pm 0.06$ & $0.6 \pm 0.09$ & $0.6 \pm 0.1$ & $0.9 \pm 0.2$ & $1.0 \pm 0.3$ & $0.4 \pm 0.4$ & GB6 J1259+5141 & a \\
\hline 130222 & 5748 & & $0.8 \pm 0.05$ & $0.7 \pm 0.06$ & $0.5 \pm 0.09$ & $0.6 \pm 0.2$ & $0.6 \pm 0.2$ & $-0.4 \pm 0.4$ & GB6 J1302+5748 & a \\
\hline 130554 & -4930 & & $1.1 \pm 0.05$ & $1.0 \pm 0.08$ & $0.8 \pm 0.09$ & $1.1 \pm 0.2$ & $1.0 \pm 0.3$ & $-0.1 \pm 0.3$ & PMN J1305-4928 & \\
\hline 131038 & 3222 & 052 & $2.5 \pm 0.05$ & $2.5 \pm 0.09$ & $2.3 \pm 0.1$ & $1.6 \pm 0.2$ & & $-0.2 \pm 0.1$ & GB6 J1310+3220 & $\mathrm{V}$ \\
\hline 131606 & -3337 & 182 & $1.7 \pm 0.06$ & $1.7 \pm 0.09$ & $1.9 \pm 0.1$ & $1.9 \pm 0.2$ & $1.6 \pm 0.4$ & $0.1 \pm 0.2$ & PMN J1316-3339 & $\mathrm{v}$ \\
\hline 132432 & -1047 & & $0.8 \pm 0.08$ & $0.8 \pm 0.1$ & $0.9 \pm 0.1$ & $1.2 \pm 0.2$ & $2.1 \pm 0.6$ & $0.5 \pm 0.4$ & PMN J1324-1049 & \\
\hline 132735 & 2213 & & $0.9 \pm 0.06$ & $0.8 \pm 0.08$ & $0.7 \pm 0.1$ & $0.4 \pm 0.2$ & $\ldots$ & $-0.4 \pm 0.5$ & GB6 J1327+2210 & $\mathrm{a}, \mathrm{v}$ \\
\hline 132901 & 3200 & 040 & $0.8 \pm 0.04$ & $0.6 \pm 0.08$ & $0.4 \pm 0.07$ & $0.4 \pm 0.2$ & $\ldots$ & $-0.9 \pm 0.5$ & & \\
\hline 133053 & 2502 & & $1.1 \pm 0.05$ & $1.0 \pm 0.07$ & $0.9 \pm 0.09$ & $0.8 \pm 0.1$ & $0.7 \pm 0.2$ & $-0.4 \pm 0.3$ & GB6 J1330+2509 & a \\
\hline 133117 & 3030 & 026 & $2.2 \pm 0.05$ & $1.8 \pm 0.09$ & $1.4 \pm 0.1$ & $1.2 \pm 0.2$ & & $-0.7 \pm 0.2$ & GB6 J1331+3030 & \\
\hline 133252 & 0200 & & $1.4 \pm 0.05$ & $1.4 \pm 0.09$ & $1.3 \pm 0.1$ & $1.2 \pm 0.2$ & $1.2 \pm 0.3$ & $-0.1 \pm 0.2$ & GB6 J1332+0200 & \\
\hline 133329 & 2723 & & $0.8 \pm 0.06$ & $0.9 \pm 0.08$ & $0.8 \pm 0.08$ & $0.7 \pm 0.1$ & $\ldots$ & $-0.1 \pm 0.4$ & GB6 J1333+2725 & a \\
\hline 133650 & -3358 & 185 & $1.9 \pm 0.06$ & $1.5 \pm 0.07$ & $1.2 \pm 0.09$ & $1.1 \pm 0.2$ & $\ldots$ & $-0.7 \pm 0.2$ & PMN J1336-3358 & \\
\hline 133740 & -1257 & 188 & $5.8 \pm 0.06$ & $6.0 \pm 0.1$ & $6.1 \pm 0.1$ & $5.7 \pm 0.2$ & $3.9 \pm 0.3$ & $0.0 \pm 0.06$ & PMN J1337-1257 & $\mathrm{V}$ \\
\hline 134400 & 6601 & & $0.7 \pm 0.07$ & $0.4 \pm 0.1$ & $0.5 \pm 0.1$ & $0.2 \pm 0.1$ & $0.9 \pm 0.2$ & $-0.2 \pm 0.4$ & GB6 J1344+6606 & a \\
\hline 134748 & 1218 & & $1.0 \pm 0.06$ & $1.1 \pm 0.09$ & $0.9 \pm 0.1$ & $0.9 \pm 0.2$ & & $-0.0 \pm 0.4$ & GB6 J1347+1217 & \\
\hline 135451 & -1041 & 197 & $1.6 \pm 0.06$ & $1.2 \pm 0.1$ & $1.2 \pm 0.2$ & $1.4 \pm 0.3$ & $0.7 \pm 0.3$ & $-0.4 \pm 0.3$ & PMN J1354-1041 & $\mathrm{v}$ \\
\hline 135556 & 7647 & & $0.7 \pm 0.06$ & $0.8 \pm 0.1$ & $0.4 \pm 0.1$ & & $\ldots$ & $-0.3 \pm 0.8$ & & d \\
\hline 135653 & -1525 & & $0.8 \pm 0.08$ & $0.9 \pm 0.2$ & $0.7 \pm 0.2$ & $0.8 \pm 0.3$ & $0.9 \pm 0.3$ & $0.0 \pm 0.5$ & PMN J1357-1527 & $\mathrm{v}$ \\
\hline 135656 & 1919 & 004 & $1.4 \pm 0.06$ & $1.6 \pm 0.09$ & $1.4 \pm 0.1$ & $1.4 \pm 0.2$ & $\ldots$ & $0.1 \pm 0.2$ & GB6 J1357+1919 & $\mathrm{v}$ \\
\hline 140853 & -0749 & 203 & $1.1 \pm 0.07$ & $1.0 \pm 0.1$ & $1.0 \pm 0.1$ & $0.6 \pm 0.2$ & $\ldots$ & $-0.3 \pm 0.4$ & 1Jy 1406-076 & \\
\hline 141125 & 5217 & & $0.9 \pm 0.05$ & $0.3 \pm 0.1$ & & $\ldots$ & $\ldots$ & $-2.8 \pm 2$ & GB6 J1411+5212 & \\
\hline 141546 & 1322 & & $0.9 \pm 0.06$ & $1.0 \pm 0.09$ & $0.8 \pm 0.09$ & $1.1 \pm 0.5$ & $0.8 \pm 0.3$ & $0.1 \pm 0.4$ & GB6 J1415+1320 & \\
\hline 141932 & 5425 & & $0.8 \pm 0.06$ & $0.8 \pm 0.09$ & $0.8 \pm 0.08$ & $1.3 \pm 0.2$ & $\ldots$ & $0.2 \pm 0.4$ & GB6 J1419+5423 & $\mathrm{a}, \mathrm{v}$ \\
\hline 141938 & 3822 & 042 & $1.1 \pm 0.04$ & $1.2 \pm 0.06$ & $1.1 \pm 0.06$ & $1.3 \pm 0.1$ & $\ldots$ & $0.2 \pm 0.2$ & GB6 J1419+3822 & $\mathrm{v}$ \\
\hline 142007 & 2704 & & $0.9 \pm 0.06$ & $1.1 \pm 0.08$ & $1.0 \pm 0.07$ & $0.8 \pm 0.1$ & $1.0 \pm 0.2$ & $0.1 \pm 0.3$ & GB6 J1419+2706 & a \\
\hline 142728 & -3302 & 193 & $1.0 \pm 0.06$ & $1.4 \pm 0.09$ & $1.7 \pm 0.1$ & $1.5 \pm 0.2$ & $\ldots$ & $0.6 \pm 0.3$ & PMN J1427-3306 & \\
\hline 142753 & -4206 & 191 & $3.1 \pm 0.05$ & $2.9 \pm 0.08$ & $2.8 \pm 0.1$ & $2.7 \pm 0.2$ & $1.9 \pm 0.3$ & $-0.2 \pm 0.1$ & PMN J1427-4206 & $\mathrm{v}$ \\
\hline 143702 & 6337 & & $0.5 \pm 0.06$ & $\ldots$ & $\ldots$ & $0.7 \pm 0.2$ & $\ldots$ & $0.3 \pm 0.7$ & GB6 J1436+6336 & a \\
\hline 144257 & 5155 & & $0.8 \pm 0.05$ & $0.9 \pm 0.07$ & $0.8 \pm 0.08$ & $0.9 \pm 0.2$ & $1.0 \pm 0.3$ & $0.1 \pm 0.3$ & GB6 J1443+5201 & \\
\hline 144655 & -1621 & & $1.0 \pm 0.06$ & $1.0 \pm 0.09$ & $0.8 \pm 0.09$ & $0.9 \pm 0.1$ & & $-0.3 \pm 0.3$ & & \\
\hline 145720 & -3536 & & $0.8 \pm 0.1$ & $0.9 \pm 0.1$ & $1.0 \pm 0.1$ & $0.9 \pm 0.2$ & $1.0 \pm 0.4$ & $0.2 \pm 0.4$ & PMN J1457-3538 & \\
\hline 145832 & 7140 & 071 & $1.3 \pm 0.07$ & $1.2 \pm 0.1$ & $0.9 \pm 0.09$ & $\ldots$ & $0.6 \pm 0.2$ & $-0.6 \pm 0.3$ & GB6 J1459+7140 & v \\
\hline 150432 & 1030 & 006 & $1.6 \pm 0.05$ & $1.5 \pm 0.09$ & $1.3 \pm 0.09$ & $0.8 \pm 0.1$ & $\ldots$ & $-0.4 \pm 0.2$ & GB6 J1504+1029 & $\mathrm{V}$ \\
\hline 150655 & -1644 & & $1.4 \pm 0.08$ & $1.3 \pm 0.2$ & $1.0 \pm 0.2$ & $0.8 \pm 0.2$ & $\ldots$ & $-0.5 \pm 0.4$ & PMN J1507-1652 & a \\
\hline 151038 & -0546 & & $1.1 \pm 0.07$ & $1.1 \pm 0.1$ & $1.1 \pm 0.1$ & $0.8 \pm 0.2$ & $\ldots$ & $-0.1 \pm 0.4$ & PMN J1510-0543 & \\
\hline 151246 & -0904 & 207 & $1.8 \pm 0.06$ & $1.7 \pm 0.1$ & $1.9 \pm 0.1$ & $1.9 \pm 0.2$ & $1.6 \pm 0.4$ & $0.0 \pm 0.2$ & 1Jy $1510-08$ & $\mathrm{v}$ \\
\hline 151349 & -1000 & & $1.3 \pm 0.06$ & $1.0 \pm 0.1$ & $0.9 \pm 0.2$ & $1.3 \pm 0.3$ & & $-0.3 \pm 0.4$ & $\ldots$ & \\
\hline 151638 & 0014 & 002 & $1.6 \pm 0.06$ & $1.8 \pm 0.09$ & $1.7 \pm 0.1$ & $1.5 \pm 0.2$ & $0.7 \pm 0.3$ & $0.0 \pm 0.2$ & GB6 J1516+0015 & \\
\hline 151744 & -2421 & 205 & $2.0 \pm 0.06$ & $2.1 \pm 0.1$ & $2.1 \pm 0.1$ & $2.0 \pm 0.2$ & $2.3 \pm 0.5$ & $0.0 \pm 0.2$ & PMN J1517-2422 & \\
\hline 154058 & 1447 & & $1.0 \pm 0.06$ & $0.8 \pm 0.09$ & $0.8 \pm 0.1$ & $0.8 \pm 0.2$ & $\ldots$ & $-0.4 \pm 0.4$ & GB6 J1540+1447 & \\
\hline 154921 & 5036 & & $0.9 \pm 0.06$ & $0.8 \pm 0.09$ & $1.0 \pm 0.1$ & $0.7 \pm 0.2$ & $0.6 \pm 0.3$ & $-0.1 \pm 0.4$ & GB6 J1549+5038 & \\
\hline 154932 & 0236 & 005 & $2.7 \pm 0.06$ & $2.9 \pm 0.1$ & $2.4 \pm 0.1$ & $2.1 \pm 0.2$ & $2.2 \pm 0.6$ & $-0.2 \pm 0.1$ & GB6 J1549+0237 & $\mathrm{v}$ \\
\hline 155039 & 0526 & 007 & $2.5 \pm 0.06$ & $2.1 \pm 0.09$ & $1.9 \pm 0.1$ & $2.0 \pm 0.2$ & $1.4 \pm 0.3$ & $-0.4 \pm 0.2$ & GB6 J1550+0527 & \\
\hline 160200 & 3329 & & $0.9 \pm 0.04$ & $0.8 \pm 0.06$ & $0.8 \pm 0.06$ & $0.5 \pm 0.1$ & $0.8 \pm 0.3$ & $-0.2 \pm 0.3$ & GB6 J1602+3326 & \\
\hline 160434 & 5718 & & $0.7 \pm 0.04$ & $0.7 \pm 0.07$ & $0.8 \pm 0.06$ & $0.5 \pm 0.1$ & $0.6 \pm 0.2$ & $-0.1 \pm 0.3$ & GB6 J1604+5714 & $\mathrm{a}$ \\
\hline 160852 & 1027 & 009 & $2.0 \pm 0.06$ & $2.0 \pm 0.1$ & $1.9 \pm 0.1$ & $1.5 \pm 0.2$ & $1.1 \pm 0.4$ & $-0.2 \pm 0.2$ & GB6 J1608+1029 & $\mathrm{v}$ \\
\hline
\end{tabular}


Table 1

(Continued)

\begin{tabular}{|c|c|c|c|c|c|c|c|c|c|c|}
\hline R.A. [hms] & Decl. [dm] & ID & $\mathrm{K}[\mathrm{Jy}]$ & $\mathrm{Ka}[\mathrm{Jy}]$ & Q [Jy] & V [Jy] & $\mathrm{W}[\mathrm{Jy}]$ & $\alpha$ & $5 \mathrm{GHz}$ ID & \\
\hline 161342 & 3412 & 023 & $4.1 \pm 0.05$ & $3.7 \pm 0.08$ & $3.4 \pm 0.08$ & $2.8 \pm 0.2$ & $1.8 \pm 0.3$ & $-0.3 \pm 0.08$ & GB6 J1613+3412 & $\mathrm{V}$ \\
\hline 161801 & -7716 & 183 & $2.4 \pm 0.05$ & $2.1 \pm 0.07$ & $1.9 \pm 0.08$ & $1.5 \pm 0.2$ & $0.9 \pm 0.2$ & $-0.4 \pm 0.1$ & PMN J1617-7717 & \\
\hline 162325 & -6817 & & $0.7 \pm 0.04$ & $0.6 \pm 0.06$ & $0.6 \pm 0.07$ & & $\ldots$ & $-0.3 \pm 0.4$ & PMN J1624-6809 & \\
\hline 162617 & 4127 & & $0.9 \pm 0.05$ & $0.8 \pm 0.08$ & $0.7 \pm 0.08$ & $0.7 \pm 0.2$ & $\ldots$ & $-0.3 \pm 0.3$ & GB6 J1625+4134 & a \\
\hline 163320 & 8226 & 076 & $1.3 \pm 0.04$ & $1.5 \pm 0.07$ & $1.5 \pm 0.08$ & $1.2 \pm 0.1$ & $0.7 \pm 0.3$ & $-0.0 \pm 0.2$ & & $\mathrm{e}$ \\
\hline 163516 & 3807 & 033 & $3.9 \pm 0.05$ & $4.3 \pm 0.08$ & $4.2 \pm 0.08$ & $3.8 \pm 0.1$ & $3.1 \pm 0.3$ & $0.1 \pm 0.07$ & GB6 J1635+3808 & $\mathrm{V}$ \\
\hline 163731 & 4713 & & $0.9 \pm 0.05$ & $1.0 \pm 0.08$ & $1.0 \pm 0.1$ & $0.9 \pm 0.1$ & $\ldots$ & $0.0 \pm 0.3$ & GB6 J1637+4717 & \\
\hline 163752 & -7714 & & $1.4 \pm 0.05$ & $0.9 \pm 0.09$ & $0.8 \pm 0.09$ & $0.7 \pm 0.1$ & - & $-0.8 \pm 0.3$ & PMN J1636-7713 & \\
\hline 163816 & 5722 & 056 & $1.3 \pm 0.04$ & $1.3 \pm 0.07$ & $1.4 \pm 0.08$ & $1.7 \pm 0.2$ & $1.0 \pm 0.3$ & $0.1 \pm 0.2$ & GB6 J1638+5720 & \\
\hline 164234 & 6854 & 069 & $1.4 \pm 0.05$ & $1.5 \pm 0.08$ & $1.5 \pm 0.09$ & $1.7 \pm 0.2$ & $1.2 \pm 0.2$ & $0.0 \pm 0.2$ & GB6 J1642+6856 & $\mathrm{a}, \mathrm{V}$ \\
\hline 164255 & 3948 & 035 & $6.5 \pm 0.05$ & $6.0 \pm 0.08$ & $5.5 \pm 0.08$ & $4.9 \pm 0.2$ & $3.9 \pm 0.3$ & $-0.3 \pm 0.05$ & GB6 J1642+3948 & V \\
\hline 165107 & 0458 & 010 & $1.6 \pm 0.07$ & $1.1 \pm 0.1$ & $1.0 \pm 0.2$ & $0.7 \pm 0.1$ & $\ldots$ & $-0.9 \pm 0.4$ & GB6 J1651+0459 & \\
\hline 165410 & 3939 & 036 & $1.2 \pm 0.05$ & $1.2 \pm 0.08$ & $0.9 \pm 0.07$ & $0.5 \pm 0.2$ & 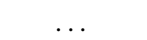 & $-0.5 \pm 0.3$ & GB6 J1653+3945 & $\mathrm{a}$ \\
\hline 165701 & 5706 & & $0.5 \pm 0.06$ & $0.6 \pm 0.09$ & $0.6 \pm 0.1$ & $0.7 \pm 0.1$ & $0.8 \pm 0.2$ & $0.4 \pm 0.4$ & GB6 J1657+5705 & \\
\hline 165726 & 4754 & & $1.1 \pm 0.04$ & $0.9 \pm 0.06$ & $0.7 \pm 0.06$ & & & $-0.6 \pm 0.3$ & & f \\
\hline 165805 & 0742 & 013 & $1.4 \pm 0.05$ & $1.5 \pm 0.07$ & $1.4 \pm 0.1$ & $1.6 \pm 0.2$ & $1.6 \pm 0.7$ & $0.1 \pm 0.2$ & GB6 J1658+0741 & $\mathrm{V}$ \\
\hline 165851 & 0513 & & $0.8 \pm 0.06$ & $0.6 \pm 0.09$ & $0.5 \pm 0.09$ & $0.4 \pm 0.2$ & & $-0.8 \pm 0.5$ & GB6 J1658+0 & \\
\hline 165952 & 6827 & & $0.2 \pm 0.06$ & $0.5 \pm 0.08$ & $0.6 \pm 0.08$ & $0.7 \pm 0.09$ & $0.6 \pm 0.1$ & $0.5 \pm 0.5$ & GB6 J1700+6 & \\
\hline 170337 & -6214 & 198 & $1.7 \pm 0.04$ & $1.7 \pm 0.07$ & $1.7 \pm 0.07$ & $1.4 \pm 0.1$ & $\ldots$ & $-0.1 \pm 0.2$ & PMN J1703-6212 & $\mathrm{v}$ \\
\hline 170737 & 0148 & & $0.8 \pm 0.06$ & $0.9 \pm 0.1$ & $0.7 \pm 0.08$ & $0.8 \pm 0.2$ & $\ldots$ & $-0.0 \pm 0.4$ & GB6 J1707+0148 & \\
\hline 171550 & 6839 & & $0.6 \pm 0.04$ & $0.6 \pm 0.06$ & $0.6 \pm 0.07$ & $\ldots$ & $0.7 \pm 0.2$ & $0.1 \pm 0.4$ & GB6 J1716+6836 & \\
\hline 172400 & -6500 & 196 & $2.3 \pm 0.05$ & $2.0 \pm 0.08$ & $1.6 \pm 0.09$ & $1.1 \pm 0.2$ & $1.2 \pm 0.3$ & $-0.6 \pm 0.2$ & PMN J1723-6500 & \\
\hline 172723 & 4530 & 043 & $0.9 \pm 0.04$ & $1.0 \pm 0.08$ & $0.8 \pm 0.07$ & $1.2 \pm 0.2$ & $1.1 \pm 0.3$ & $0.1 \pm 0.3$ & GB6 J1727+4530 & V \\
\hline 173416 & 3857 & 038 & $1.2 \pm 0.05$ & $1.3 \pm 0.08$ & $1.2 \pm 0.09$ & $1.3 \pm 0.2$ & $\ldots$ & $0.1 \pm 0.2$ & GB6 J1734+3857 & \\
\hline 173612 & -7934 & 186 & $1.0 \pm 0.04$ & $1.1 \pm 0.07$ & $1.2 \pm 0.07$ & $0.9 \pm 0.1$ & $\ldots$ & $0.1 \pm 0.2$ & PMN J1733-7935 & \\
\hline 173826 & 5015 & & $0.8 \pm 0.04$ & $0.5 \pm 0.08$ & $0.6 \pm 0.08$ & $0.5 \pm 0.1$ & $\ldots$ & $-0.5 \pm 0.4$ & & \\
\hline 174011 & 4740 & & $0.8 \pm 0.05$ & $0.8 \pm 0.06$ & $0.9 \pm 0.08$ & $0.8 \pm 0.2$ & $\ldots$ & $0.1 \pm 0.3$ & GB6 J1739+4738 & \\
\hline 174034 & 5212 & 048 & $1.2 \pm 0.04$ & $1.2 \pm 0.07$ & $1.3 \pm 0.1$ & $1.2 \pm 0.2$ & $0.8 \pm 0.3$ & $-0.0 \pm 0.2$ & GB6 J1740+5211 & \\
\hline 174855 & 7006 & 068 & $0.6 \pm 0.03$ & $0.7 \pm 0.06$ & $0.8 \pm 0.06$ & $1.0 \pm 0.1$ & $0.7 \pm 0.1$ & $0.4 \pm 0.2$ & GB6 J1748+7005 & \\
\hline 175324 & 4408 & & $0.7 \pm 0.06$ & $0.6 \pm 0.1$ & $0.8 \pm 0.09$ & & $1.1 \pm 0.3$ & $0.3 \pm 0.4$ & GB6 J1753+4410 & \\
\hline 175333 & 2848 & 022 & $2.1 \pm 0.05$ & $1.9 \pm 0.07$ & $2.1 \pm 0.08$ & $2.2 \pm 0.2$ & $1.6 \pm 0.7$ & $-0.0 \pm 0.1$ & GB6 J1753+2847 & $\mathrm{v}$ \\
\hline 175858 & 6632 & 064 & $0.6 \pm 0.02$ & $0.6 \pm 0.03$ & $0.6 \pm 0.05$ & $0.4 \pm 0.1$ & $\ldots$ & $-0.1 \pm 0.2$ & GB6 J1758+6638 & a \\
\hline 175950 & 3852 & & $0.9 \pm 0.05$ & $0.8 \pm 0.07$ & $0.7 \pm 0.1$ & $\ldots$ & $\ldots$ & $-0.4 \pm 0.5$ & GB6 J1800+3848 & a \\
\hline 180027 & 7827 & 072 & $1.8 \pm 0.05$ & $1.7 \pm 0.07$ & $1.6 \pm 0.08$ & $1.5 \pm 0.2$ & $0.9 \pm 0.2$ & $-0.3 \pm 0.2$ & 1Jy $1803+78$ & $\mathrm{v}$ \\
\hline 180132 & 4404 & & $1.2 \pm 0.04$ & $1.4 \pm 0.07$ & $1.6 \pm 0.1$ & $1.5 \pm 0.2$ & $1.1 \pm 0.2$ & $0.2 \pm 0.2$ & GB6 J1801+4404 & $\mathrm{v}$ \\
\hline 180300 & -6507 & 199 & $1.2 \pm 0.05$ & $1.1 \pm 0.08$ & $1.3 \pm 0.09$ & $1.1 \pm 0.2$ & $0.9 \pm 0.2$ & $-0.0 \pm 0.2$ & PMN J1803-6507 & $\mathrm{v}$ \\
\hline 180647 & 6949 & 067 & $1.4 \pm 0.03$ & $1.4 \pm 0.06$ & $1.2 \pm 0.07$ & $1.4 \pm 0.1$ & $1.2 \pm 0.3$ & $-0.1 \pm 0.1$ & GB6 J1806+6949 & $\mathrm{v}$ \\
\hline 180832 & 5658 & & $0.6 \pm 0.05$ & $0.7 \pm 0.06$ & $0.8 \pm 0.06$ & $0.7 \pm 0.09$ & $\ldots$ & $0.3 \pm 0.3$ & GB6 J1808+5709 & $\mathrm{a}$ \\
\hline 181957 & -5521 & & $0.9 \pm 0.07$ & $0.5 \pm 0.2$ & $0.6 \pm 0.1$ & $\ldots$ & $\ldots$ & $-0.8 \pm 0.8$ & PMN J1819-5521 & \\
\hline 182003 & -6343 & 200 & $1.7 \pm 0.05$ & $1.5 \pm 0.08$ & $1.2 \pm 0.09$ & $1.2 \pm 0.2$ & $1.2 \pm 0.2$ & $-0.3 \pm 0.2$ & PMN J1819-6345 & \\
\hline 182409 & 5650 & 053 & $1.5 \pm 0.04$ & $1.3 \pm 0.07$ & $1.3 \pm 0.09$ & $1.4 \pm 0.2$ & $0.8 \pm 0.2$ & $-0.3 \pm 0.2$ & GB6 J1824+5650 & \\
\hline 182537 & 6737 & & & $\ldots$ & $0.3 \pm 0.09$ & $0.6 \pm 0.1$ & $0.6 \pm 0.1$ & $0.7 \pm 0.8$ & & \\
\hline 182942 & 4845 & 046 & $2.8 \pm 0.04$ & $2.8 \pm 0.07$ & $2.6 \pm 0.08$ & $2.0 \pm 0.1$ & $1.3 \pm 0.2$ & $-0.2 \pm 0.1$ & GB6 J1829+4844 & $\mathrm{v}$ \\
\hline 183241 & 6844 & & $\ldots$ & $\ldots$ & $0.4 \pm 0.07$ & $0.7 \pm 0.06$ & $0.7 \pm 0.1$ & $0.8 \pm 0.8$ & GB6 J1832+6848 & \\
\hline 183421 & -5854 & & $1.1 \pm 0.04$ & $1.1 \pm 0.07$ & $1.2 \pm 0.08$ & $0.9 \pm 0.2$ & $\ldots$ & $0.1 \pm 0.3$ & PMN J1834-5856 & \\
\hline 183503 & 3245 & & $0.8 \pm 0.05$ & $0.8 \pm 0.07$ & $0.7 \pm 0.07$ & $0.5 \pm 0.2$ & $0.7 \pm 0.2$ & $-0.2 \pm 0.3$ & GB6 J1835+3241 & \\
\hline 183723 & -7106 & 192 & $1.9 \pm 0.04$ & $1.7 \pm 0.06$ & $1.5 \pm 0.06$ & $1.2 \pm 0.1$ & $\ldots$ & $-0.4 \pm 0.1$ & PMN J1837-7108 & \\
\hline 184049 & 7946 & 073 & $1.3 \pm 0.04$ & $0.9 \pm 0.08$ & $0.7 \pm 0.1$ & 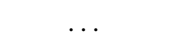 & $\ldots$ & $-1.0 \pm 0.4$ & 1Jy $1845+79$ & \\
\hline 184252 & 6808 & 066 & $1.1 \pm 0.03$ & $1.2 \pm 0.05$ & $1.2 \pm 0.05$ & $1.0 \pm 0.08$ & $0.6 \pm 0.2$ & $-0.0 \pm 0.1$ & GB6 J1842+6809 & a \\
\hline 184840 & 3223 & & $0.7 \pm 0.05$ & $0.8 \pm 0.1$ & $0.5 \pm 0.1$ & $\ldots$ & $\ldots$ & $-0.3 \pm 0.8$ & GB6 J1848+3219 & \\
\hline 184938 & 6705 & 065 & $1.2 \pm 0.04$ & $1.4 \pm 0.06$ & $1.4 \pm 0.05$ & $1.2 \pm 0.1$ & $1.4 \pm 0.2$ & $0.1 \pm 0.1$ & GB6 J1849+6705 & $\mathrm{a}, \mathrm{V}$ \\
\hline 185045 & 2823 & 028 & $1.5 \pm 0.04$ & $1.1 \pm 0.07$ & $0.9 \pm 0.05$ & $0.6 \pm 0.1$ & $\ldots$ & $-0.9 \pm 0.2$ & GB6 J1850+2825 & \\
\hline 190253 & 3153 & 034 & $1.3 \pm 0.04$ & $1.1 \pm 0.06$ & $0.8 \pm 0.07$ & $0.4 \pm 0.2$ & $\cdots$ & $-0.8 \pm 0.3$ & GB6 J1902+3159 & \\
\hline 191556 & -8000 & & $0.7 \pm 0.04$ & $0.4 \pm 0.07$ & $0.5 \pm 0.07$ & $\ldots$ & $\ldots$ & $-0.9 \pm 0.5$ & PMN J1918-7957 & \\
\hline 192330 & -2105 & 008 & $2.3 \pm 0.06$ & $2.5 \pm 0.1$ & $2.5 \pm 0.1$ & $2.6 \pm 0.2$ & $2.0 \pm 0.4$ & $0.1 \pm 0.1$ & PMN J1923-2104 & $\mathrm{v}$ \\
\hline 192451 & -2914 & & $12.3 \pm 0.06$ & $12.0 \pm 0.1$ & $11.4 \pm 0.1$ & $10.9 \pm 0.2$ & $7.6 \pm 0.4$ & $-0.1 \pm 0.03$ & PMN J1924-2914 & \\
\hline 192736 & 6119 & 059 & $1.0 \pm 0.04$ & $1.0 \pm 0.07$ & $1.0 \pm 0.08$ & $0.7 \pm 0.1$ & $0.6 \pm 0.2$ & $-0.2 \pm 0.3$ & GB6 J1927+6117 & \\
\hline 192742 & 7357 & 070 & $3.5 \pm 0.04$ & $3.2 \pm 0.06$ & $2.8 \pm 0.07$ & $2.7 \pm 0.1$ & $1.3 \pm 0.3$ & $-0.3 \pm 0.07$ & GB6 J1927+7357 & V \\
\hline 193707 & -3957 & & $1.0 \pm 0.07$ & $1.3 \pm 0.1$ & $1.3 \pm 0.1$ & $1.4 \pm 0.2$ & $\ldots$ & $0.3 \pm 0.3$ & PMN J1937-3957 & $\mathrm{V}$ \\
\hline 193815 & -6344 & & $0.9 \pm 0.05$ & $0.7 \pm 0.07$ & $0.6 \pm 0.08$ & 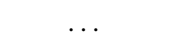 & $\cdots$ & $-0.6 \pm 0.4$ & PMN J1939-6342 & a \\
\hline 193924 & -1525 & & $1.0 \pm 0.07$ & $1.0 \pm 0.09$ & $1.0 \pm 0.09$ & $0.6 \pm 0.2$ & & $-0.1 \pm 0.4$ & PMN J1939-1525 & \\
\hline 195128 & 6749 & & $0.7 \pm 0.04$ & $0.9 \pm 0.05$ & $0.7 \pm 0.05$ & $0.7 \pm 0.08$ & $0.7 \pm 0.2$ & $0.1 \pm 0.2$ & GB6 J1951+6743 & \\
\hline 195219 & 0233 & & $0.8 \pm 0.07$ & $0.6 \pm 0.09$ & $0.6 \pm 0.08$ & $0.9 \pm 0.2$ & & $-0.3 \pm 0.5$ & GB6 J1952+0230 & \\
\hline 195546 & 5139 & 051 & $0.8 \pm 0.05$ & $0.9 \pm 0.1$ & $0.8 \pm 0.09$ & $\ldots$ & & $0.1 \pm 0.4$ & GB6 J1955+5131 & \\
\hline 195802 & -3845 & 003 & $3.4 \pm 0.06$ & $3.5 \pm 0.09$ & $3.1 \pm 0.1$ & $2.7 \pm 0.2$ & $1.9 \pm 0.5$ & $-0.1 \pm 0.1$ & PMN J1957-3845 & $\mathrm{v}$ \\
\hline 200058 & -1749 & 011 & $2.0 \pm 0.07$ & $1.9 \pm 0.09$ & $1.8 \pm 0.1$ & $1.8 \pm 0.2$ & $2.0 \pm 0.8$ & $-0.1 \pm 0.2$ & PMN J2000-1748 & $\mathrm{v}$ \\
\hline
\end{tabular}


Table 1

(Continued)

\begin{tabular}{|c|c|c|c|c|c|c|c|c|c|c|}
\hline R.A. [hms] & Decl. [dm] & ID & $\mathrm{K}[\mathrm{Jy}]$ & $\mathrm{Ka}[\mathrm{Jy}]$ & $\mathrm{Q}[\mathrm{Jy}]$ & $\mathrm{V}[\mathrm{Jy}]$ & $\mathrm{W}$ [Jy] & $\alpha$ & $5 \mathrm{GHz}$ ID & \\
\hline 200543 & 7755 & & $0.8 \pm 0.05$ & $0.7 \pm 0.1$ & $0.8 \pm 0.1$ & $0.8 \pm 0.2$ & $0.9 \pm 0.2$ & $0.0 \pm 0.3$ & 1Jy $2007+77$ & $\mathrm{v}$ \\
\hline 200821 & 6612 & & $0.7 \pm 0.03$ & $0.5 \pm 0.05$ & $0.5 \pm 0.05$ & $0.6 \pm 0.2$ & $\ldots$ & $-0.5 \pm 0.3$ & GB6 J2007+6607 & \\
\hline 201003 & 7231 & & $0.7 \pm 0.06$ & $0.6 \pm 0.08$ & $1.0 \pm 0.07$ & $1.0 \pm 0.2$ & $0.7 \pm 0.2$ & $0.4 \pm 0.3$ & GB6 J2009+7229 & \\
\hline 201119 & -1547 & 014 & $1.6 \pm 0.05$ & $1.5 \pm 0.1$ & $1.5 \pm 0.2$ & $1.2 \pm 0.3$ & $\ldots$ & $-0.2 \pm 0.3$ & PMN J2011-1546 & \\
\hline 202230 & 6136 & 063 & $1.6 \pm 0.05$ & $1.4 \pm 0.07$ & $1.2 \pm 0.07$ & $0.7 \pm 0.1$ & $\ldots$ & $-0.5 \pm 0.2$ & GB6 J2022+6137 & \\
\hline 202338 & 5426 & & $0.7 \pm 0.07$ & $0.9 \pm 0.07$ & $0.8 \pm 0.08$ & $0.8 \pm 0.1$ & $\ldots$ & $0.1 \pm 0.4$ & GB6 J2023+5427 & \\
\hline 202431 & 1712 & 031 & $0.9 \pm 0.04$ & $1.0 \pm 0.08$ & $0.9 \pm 0.09$ & $0.8 \pm 0.1$ & $0.8 \pm 0.3$ & $-0.1 \pm 0.3$ & GB6 J2024+1718 & $\mathrm{a}$ \\
\hline 203454 & -6845 & 194 & $0.7 \pm 0.05$ & $0.8 \pm 0.08$ & $0.8 \pm 0.08$ & $0.8 \pm 0.09$ & $\ldots$ & $0.2 \pm 0.3$ & PMN J2035-6846 & \\
\hline 203520 & 1055 & & $0.6 \pm 0.06$ & $1.1 \pm 0.1$ & $0.7 \pm 0.1$ & $0.9 \pm 0.2$ & $1.0 \pm 0.3$ & $0.3 \pm 0.3$ & GB6 J2035+1055 & \\
\hline 205611 & -4716 & 208 & $2.2 \pm 0.05$ & $2.5 \pm 0.08$ & $2.4 \pm 0.1$ & $2.2 \pm 0.2$ & $1.7 \pm 0.4$ & $0.1 \pm 0.1$ & PMN J2056-4714 & $\mathrm{V}$ \\
\hline 210137 & 0344 & & $1.2 \pm 0.05$ & $1.1 \pm 0.08$ & $1.0 \pm 0.2$ & $1.0 \pm 0.2$ & $0.9 \pm 0.3$ & $-0.2 \pm 0.3$ & GB6 J2101+0341 & $\mathrm{v}$ \\
\hline 210931 & -4113 & 001 & $1.5 \pm 0.06$ & $1.6 \pm 0.1$ & $1.2 \pm 0.1$ & $1.1 \pm 0.2$ & $1.0 \pm 0.3$ & $-0.3 \pm 0.2$ & PMN J2109-4110 & \\
\hline 210939 & 3537 & 049 & $0.9 \pm 0.06$ & $0.7 \pm 0.08$ & $0.6 \pm 0.07$ & $0.8 \pm 0.2$ & $\ldots$ & $-0.4 \pm 0.4$ & GB6 J2109+3532 & $\mathrm{a}, \mathrm{v}$ \\
\hline 212342 & 0536 & 027 & $2.2 \pm 0.06$ & $1.8 \pm 0.1$ & $1.8 \pm 0.1$ & $1.3 \pm 0.2$ & $\ldots$ & $-0.4 \pm 0.2$ & GB6 J2123+0535 & $\mathrm{v}$ \\
\hline 212410 & 2507 & & $0.8 \pm 0.06$ & $0.6 \pm 0.09$ & $0.4 \pm 0.08$ & $0.5 \pm 0.2$ & $\ldots$ & $-0.9 \pm 0.6$ & GB6 J2123+2504 & \\
\hline 213132 & -1207 & 017 & $2.7 \pm 0.06$ & $2.4 \pm 0.1$ & $2.4 \pm 0.1$ & $1.7 \pm 0.2$ & $1.6 \pm 0.5$ & $-0.3 \pm 0.1$ & PMN J2131-1207 & $\mathrm{v}$ \\
\hline 213408 & -0154 & 020 & $2.0 \pm 0.06$ & $1.9 \pm 0.1$ & $1.7 \pm 0.1$ & $1.6 \pm 0.2$ & $1.5 \pm 0.4$ & $-0.2 \pm 0.2$ & PMN J2134-0153 & \\
\hline 213637 & 0041 & 025 & $4.4 \pm 0.06$ & $3.5 \pm 0.1$ & $3.0 \pm 0.1$ & $1.4 \pm 0.2$ & $1.4 \pm 0.3$ & $-0.7 \pm 0.1$ & GB6 J2136+0041 & \\
\hline 213918 & 1425 & 041 & $2.2 \pm 0.05$ & $2.0 \pm 0.08$ & $1.9 \pm 0.09$ & $1.3 \pm 0.2$ & $1.0 \pm 0.2$ & $-0.3 \pm 0.2$ & GB6 J2139+1423 & $\mathrm{v}$ \\
\hline 214326 & 1741 & 044 & $1.2 \pm 0.05$ & $1.3 \pm 0.07$ & $1.0 \pm 0.09$ & $0.8 \pm 0.2$ & $\ldots$ & $-0.1 \pm 0.3$ & GB6 J2143+1743 & \\
\hline 214805 & 0657 & 037 & $8.0 \pm 0.06$ & $7.7 \pm 0.09$ & $7.5 \pm 0.1$ & $6.5 \pm 0.2$ & $5.3 \pm 0.5$ & $-0.2 \pm 0.05$ & GB6 J2148+0657 & $\mathrm{v}$ \\
\hline 214846 & -7758 & 184 & $1.6 \pm 0.04$ & $1.4 \pm 0.07$ & $1.2 \pm 0.07$ & $0.7 \pm 0.1$ & $\ldots$ & $-0.5 \pm 0.2$ & PMN J2146-7755 & \\
\hline 215147 & -3027 & & $1.3 \pm 0.06$ & $1.4 \pm 0.1$ & $1.4 \pm 0.1$ & $1.6 \pm 0.2$ & $\ldots$ & $0.2 \pm 0.2$ & PMN J2151-3028 & $\mathrm{v}$ \\
\hline 215705 & -6942 & 190 & $3.6 \pm 0.05$ & $2.9 \pm 0.08$ & $2.6 \pm 0.08$ & $2.1 \pm 0.2$ & $1.5 \pm 0.4$ & $-0.6 \pm 0.1$ & PMN J2157-6941 & \\
\hline 215807 & -1501 & 018 & $2.1 \pm 0.07$ & $1.8 \pm 0.09$ & $1.8 \pm 0.1$ & $1.4 \pm 0.3$ & $0.6 \pm 0.3$ & $-0.4 \pm 0.2$ & PMN J2158-1501 & \\
\hline 220250 & 4217 & 058 & $3.4 \pm 0.05$ & $3.5 \pm 0.07$ & $3.6 \pm 0.07$ & $3.3 \pm 0.2$ & $\ldots$ & $0.0 \pm 0.08$ & GB6 J2202+4216 & $\mathrm{V}$ \\
\hline 220319 & 3146 & 054 & $2.7 \pm 0.05$ & $2.4 \pm 0.08$ & $2.1 \pm 0.1$ & $1.7 \pm 0.2$ & $1.6 \pm 0.4$ & $-0.4 \pm 0.1$ & GB6 J2203+3145 & $\mathrm{v}$ \\
\hline 220325 & 1723 & 045 & $1.5 \pm 0.06$ & $1.6 \pm 0.09$ & $1.6 \pm 0.1$ & $1.5 \pm 0.2$ & $\ldots$ & $0.1 \pm 0.2$ & GB6 J2203+1725 & \\
\hline 220613 & -1838 & 016 & $1.8 \pm 0.06$ & $1.6 \pm 0.09$ & $1.2 \pm 0.1$ & $1.1 \pm 0.2$ & $\ldots$ & $-0.5 \pm 0.2$ & PMN J2206-1835 & \\
\hline 220712 & -5348 & & $1.0 \pm 0.05$ & $0.8 \pm 0.07$ & $0.7 \pm 0.1$ & $0.4 \pm 0.1$ & $\ldots$ & $-0.6 \pm 0.4$ & PMN J2207-5346 & \\
\hline 221137 & 2352 & 050 & $1.3 \pm 0.06$ & $1.5 \pm 0.09$ & $1.4 \pm 0.08$ & $1.0 \pm 0.1$ & $1.1 \pm 0.3$ & $-0.1 \pm 0.2$ & GB6 J2212+2355 & $\mathrm{v}$ \\
\hline 221259 & -2524 & & $0.9 \pm 0.07$ & $0.7 \pm 0.1$ & $0.6 \pm 0.1$ & $0.8 \pm 0.1$ & $\ldots$ & $-0.2 \pm 0.4$ & PMN J2213-2529 & a \\
\hline 221852 & -0335 & 030 & $2.3 \pm 0.06$ & $2.0 \pm 0.1$ & $1.9 \pm 0.1$ & $1.6 \pm 0.2$ & $\ldots$ & $-0.4 \pm 0.2$ & PMN J2218-0335 & $\mathrm{v}$ \\
\hline 222538 & 2119 & & $0.8 \pm 0.06$ & $1.0 \pm 0.09$ & $0.9 \pm 0.09$ & $0.6 \pm 0.2$ & $0.6 \pm 0.2$ & $0.1 \pm 0.4$ & GB6 J2225+2118 & \\
\hline 222546 & -0455 & 029 & $5.2 \pm 0.06$ & $4.9 \pm 0.1$ & $4.3 \pm 0.1$ & $4.1 \pm 0.2$ & $3.6 \pm 0.7$ & $-0.2 \pm 0.08$ & PMN J2225-0457 & $\mathrm{V}$ \\
\hline 222942 & -0833 & 024 & $1.9 \pm 0.07$ & $2.3 \pm 0.1$ & $2.2 \pm 0.1$ & $2.9 \pm 0.2$ & $2.1 \pm 0.5$ & $0.3 \pm 0.2$ & PMN J2229-0832 & $\mathrm{V}$ \\
\hline 222947 & -2050 & & $0.9 \pm 0.06$ & $0.8 \pm 0.09$ & $0.9 \pm 0.1$ & $1.0 \pm 0.2$ & $1.0 \pm 0.3$ & $0.1 \pm 0.3$ & PMN J2229-2049 & \\
\hline 223237 & 1144 & 047 & $3.4 \pm 0.06$ & $4.0 \pm 0.1$ & $4.1 \pm 0.1$ & $4.5 \pm 0.2$ & $4.0 \pm 0.3$ & $0.2 \pm 0.08$ & GB6 J2232+1143 & $\mathrm{v}$ \\
\hline 223513 & -4834 & 206 & $2.0 \pm 0.05$ & $2.2 \pm 0.08$ & $2.1 \pm 0.1$ & $2.0 \pm 0.2$ & $1.6 \pm 0.4$ & $0.1 \pm 0.1$ & PMN J2235-4835 & $\mathrm{v}$ \\
\hline 223623 & 2824 & 057 & $1.1 \pm 0.07$ & $1.2 \pm 0.08$ & $1.2 \pm 0.1$ & $1.2 \pm 0.1$ & $\ldots$ & $0.1 \pm 0.3$ & GB6 J2236+2828 & \\
\hline 223933 & -5701 & 201 & $1.2 \pm 0.04$ & $1.4 \pm 0.05$ & $1 \pm 0.07$ & $0.9 \pm 0.1$ & $1.5 \pm 0.7$ & $-0.0 \pm 0.2$ & PMN J2239-5701 & \\
\hline 224613 & -1208 & 021 & $1.8 \pm 0.06$ & $1.8 \pm 0.1$ & $1.7 \pm 0.2$ & $1.2 \pm 0.3$ & $\ldots$ & $-0.2 \pm 0.3$ & PMN J2246-1206 & $\mathrm{V}$ \\
\hline 225400 & 1608 & 055 & $7.4 \pm 0.06$ & $7.5 \pm 0.1$ & $7.5 \pm 0.1$ & $7.6 \pm 0.2$ & $7.2 \pm 0.4$ & $0.0 \pm 0.04$ & GB6 J2253+1608 & $\mathrm{V}$ \\
\hline 225544 & 4201 & & $1.0 \pm 0.04$ & $0.7 \pm 0.06$ & $0.6 \pm 0.08$ & $\ldots$ & $\ldots$ & $-0.8 \pm 0.4$ & GB6 J2255+4202 & \\
\hline 225629 & -2014 & 019 & $0.8 \pm 0.05$ & $0.7 \pm 0.08$ & $0.8 \pm 0.09$ & $0.5 \pm 0.2$ & $\ldots$ & $-0.3 \pm 0.4$ & PMN J2256-2011 & \\
\hline 225806 & -2757 & 012 & $5.2 \pm 0.06$ & $5.2 \pm 0.09$ & $5.0 \pm 0.1$ & $4.4 \pm 0.2$ & $3.6 \pm 0.4$ & $-0.1 \pm 0.07$ & PMN J2258-2758 & $\mathrm{V}$ \\
\hline 230244 & -6808 & & $0.6 \pm 0.07$ & $0.5 \pm 0.08$ & $0.5 \pm 0.1$ & $\ldots$ & $\ldots$ & $-0.6 \pm 0.8$ & PMN J2303-6807 & a \\
\hline 231549 & -5018 & 204 & $1.1 \pm 0.04$ & $1.1 \pm 0.06$ & $0.9 \pm 0.1$ & $0.9 \pm 0.1$ & $\ldots$ & $-0.3 \pm 0.3$ & PMN J2315-5018 & $\mathrm{v}$ \\
\hline 232233 & 4448 & & $0.8 \pm 0.03$ & $0.9 \pm 0.05$ & $0.8 \pm 0.07$ & $0.6 \pm 0.1$ & $0.5 \pm 0.3$ & $-0.1 \pm 0.3$ & GB6 J2322+4445 & a \\
\hline 232248 & 5105 & & $0.9 \pm 0.05$ & $0.8 \pm 0.09$ & $0.7 \pm 0.08$ & $0.5 \pm 0.1$ & $\ldots$ & $-0.4 \pm 0.3$ & GB6 J2322+5057 & a \\
\hline 232738 & 0937 & & $0.8 \pm 0.07$ & $1.2 \pm 0.1$ & $1.1 \pm 0.1$ & $0.7 \pm 0.2$ & $\cdots$ & $0.3 \pm 0.4$ & GB6 J2327+0940 & a \\
\hline 232904 & -4733 & & $1.3 \pm 0.04$ & $1.0 \pm 0.08$ & $1.2 \pm 0.1$ & $0.8 \pm 0.1$ & $0.9 \pm 0.2$ & $-0.3 \pm 0.2$ & PMN J2329-4730 & $\mathrm{v}$ \\
\hline 233022 & 3348 & & $0.8 \pm 0.06$ & $0.8 \pm 0.09$ & $0.7 \pm 0.09$ & $0.8 \pm 0.2$ & $\ldots$ & $0.0 \pm 0.4$ & GB6 J2330+3348 & a \\
\hline 233044 & 1056 & & $1.0 \pm 0.05$ & $1.0 \pm 0.08$ & $0.9 \pm 0.08$ & $1.0 \pm 0.2$ & $\ldots$ & $-0.1 \pm 0.3$ & GB6 J2330+1100 & \\
\hline 233122 & -1558 & 032 & $1.1 \pm 0.07$ & $0.9 \pm 0.1$ & $0.8 \pm 0.2$ & $0.8 \pm 0.2$ & $0.8 \pm 0.4$ & $-0.4 \pm 0.4$ & PMN J2331-1556 & \\
\hline 233345 & -2340 & & $0.9 \pm 0.06$ & $0.9 \pm 0.08$ & $1.0 \pm 0.09$ & $1.1 \pm 0.3$ & $0.7 \pm 0.3$ & $0.1 \pm 0.3$ & PMN J2333-2343 & a \\
\hline 233410 & 0734 & & $1.1 \pm 0.07$ & $1.0 \pm 0.08$ & $1.0 \pm 0.09$ & $1.3 \pm 0.2$ & $\ldots$ & $-0.0 \pm 0.3$ & GB6 J2334+0736 & \\
\hline 233458 & -0129 & & $0.6 \pm 0.06$ & $1.1 \pm 0.1$ & $1.0 \pm 0.1$ & $0.7 \pm 0.2$ & $\cdots$ & $0.7 \pm 0.5$ & PMN J2335-0131 & \\
\hline 233527 & -5243 & 195 & $1.2 \pm 0.04$ & $0.8 \pm 0.05$ & $0.7 \pm 0.09$ & $0.6 \pm 0.1$ & $\cdots$ & $-1.0 \pm 0.3$ & PMN J2336-5236 & $\mathrm{a}, \mathrm{v}$ \\
\hline 234646 & 0929 & & $1.2 \pm 0.06$ & $1.1 \pm 0.07$ & $0.8 \pm 0.1$ & $0.6 \pm 0.2$ & & $-0.4 \pm 0.4$ & GB6 J2346+0930 & a \\
\hline 234814 & -4931 & & $0.7 \pm 0.06$ & $0.7 \pm 0.07$ & $0.7 \pm 0.07$ & & $\ldots$ & $0.1 \pm 0.5$ & $\ldots$ & \\
\hline 234816 & -1630 & 039 & $1.8 \pm 0.06$ & $1.8 \pm 0.1$ & $1.9 \pm 0.1$ & $1.5 \pm 0.2$ & $1.0 \pm 0.3$ & $-0.1 \pm 0.2$ & PMN J2348-1631 & \\
\hline 234932 & 3846 & & $0.8 \pm 0.06$ & $0.7 \pm 0.1$ & & $\ldots$ & $\ldots$ & $-0.3 \pm 1$ & GB6 J2349+3849 & a \\
\hline 235422 & 4550 & 074 & $1.6 \pm 0.05$ & $1.2 \pm 0.07$ & $1.2 \pm 0.1$ & $1.1 \pm 0.2$ & $0.9 \pm 0.2$ & $-0.4 \pm 0.2$ & GB6 J2354+4553 & \\
\hline 235459 & 8152 & & $0.8 \pm 0.04$ & $0.8 \pm 0.1$ & $0.7 \pm 0.09$ & $1.3 \pm 0.2$ & $\ldots$ & $0.1 \pm 0.3$ & & \\
\hline 235611 & 4953 & 075 & $0.9 \pm 0.03$ & $0.8 \pm 0.05$ & $0.6 \pm 0.07$ & $0.4 \pm 0.1$ & $\ldots$ & $-0.4 \pm 0.3$ & GB6 J2355+4950 & \\
\hline
\end{tabular}


Table 1

(Continued)

\begin{tabular}{|c|c|c|c|c|c|c|c|c|c|}
\hline R.A. [hms] & Decl. $[\mathrm{dm}]$ & ID & $\mathrm{K}[\mathrm{Jy}]$ & $\mathrm{Ka}[\mathrm{Jy}]$ & $\mathrm{Q}[\mathrm{Jy}]$ & $\mathrm{V}[\mathrm{Jy}]$ & $\mathrm{W}[\mathrm{Jy}]$ & $\alpha$ & $5 \mathrm{GHz}$ ID \\
\hline 235751 & -5314 & 189 & $1.3 \pm 0.04$ & $1.1 \pm 0.08$ & $1.1 \pm 0.1$ & $1.1 \pm 0.1$ & $0.8 \pm 0.3$ & $-0.2 \pm 0.2$ & PMN J2357-5311 \\
\hline 235804 & -1014 & & $1.1 \pm 0.06$ & $1.3 \pm 0.07$ & $1.2 \pm 0.08$ & $1.2 \pm 0.2$ & $0.9 \pm 0.4$ & $0.1 \pm 0.3$ & PMN J2358-1020 \\
\hline 235853 & -6050 & 187 & $1.9 \pm 0.05$ & $1.4 \pm 0.07$ & $1.2 \pm 0.06$ & $1.1 \pm 0.1$ & $\ldots$ & $-0.7 \pm 0.2$ & PMN J2358-6054 \\
\hline
\end{tabular}

Notes.

a Indicates the source has multiple possible identifications.

b Source J0322-3711 (Fornax A) is extended, and the fluxes listed were obtained by aperture photometry.

c Source J0519-0539 is a blend of the Lynds Bright Nebulae LBN 207.65-23.11 and LBN 207.29-22.66.

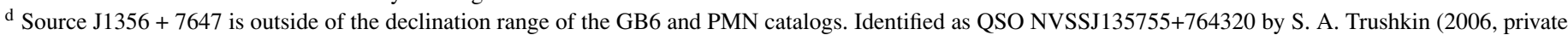
communication).

e Source J1633 + 8226 is outside of the declination range of the GB6 and PMN catalogs. It was identified as NGC 6251 by Trushkin (2003).

f Source J1657 + 4754 is identified as QSO GB6J1658 + 4737 by S. A. Trushkin (2006, private communication). Offset from the WMAP position is 18.1 arcmin.

v Probable variable: $\chi^{2}>37.6$.

V Variable: $\chi^{2}>100$.

\section{FLUX VARIABILITY OVER FIVE YEARS}

An analysis of the variability of the WMAP point sources has been performed by forming fluxes from the individual year maps. It is possible to measure the variability of a source without any noise contribution from the CMB by subtracting the five-year average map from each individual year. The fit of a Gaussian beam plus planar baseline to this difference map then gives a $\Delta F_{i}$ for the $i$ th year, and the flux for the $i$ th year is then given by $F_{i}=\langle F\rangle+\Delta F_{i}$ where the five-year average flux is $\langle F\rangle$.

There are 25 data points for a source detected in all five bands, and fitting an arbitrary spectrum that is constant in time leaves 20 degrees of freedom. 137 of the 390 sources in Table 1 give $\chi^{2}>37.6$ relative to this fit and thus are variable at greater than $99 \%$ confidence. Sources with $\chi^{2}>37.6$ are flagged with a "v" in the notes column of Table 1 . The 54 sources with $\chi^{2}>100$ have a "V" in the notes column. These are generally the brighter sources which have smaller relative flux errors, allowing a better detection of variability. The five band lightcurves for the 15 sources with $\chi^{2}>450$ are plotted in Figure 4. The median rms variability of the Q-band fluxes among the 25 brightest Q-band sources is $23 \%$, after allowing for the flux variations due to radiometer noise.

It is clear from Figure 4 that most of the variability involves the entire spectrum of a source moving up and down together, at least on the one-year time resolution of this analysis. The full table of year-by-year and band-by-band fluxes for WMAP sources will be available on LAMBDA.

\section{POLARIZATION}

In general the WMAP detected point sources are not strongly polarized. Of the 390 sources in Table 3, only five have polarizations greater than $4 \sigma$ in two or more bands. These sources are listed in Table 3. In order to assess the average polarization of the sources, the square of the polarized flux, evaluated as $Q^{2}+U^{2}-\sigma_{Q}^{2}-\sigma_{U}^{2}$, was fit to the form $p^{2} I^{2}$. This gave mean polarization percentages of $p=2.9,2.2,1.9,<3.4$, and $<8.5 \%$ in $\mathrm{K}, \mathrm{Ka}, \mathrm{Q}, \mathrm{V}$, and $\mathrm{W}$. For the $\mathrm{V}$ and $\mathrm{W}$ bands $2 \sigma$ upper limits on the mean polarization percentage are given.

\section{EFFECT ON THE POWER SPECTRUM}

Uncorrelated point sources contribute a power spectrum $C_{\ell}=$ const to the power spectrum. Since one has to divide by the beam function $b_{\ell}^{2}$ and multiply by $\ell(\ell+1) / 2 \pi$ to put this on the usual angular power spectrum plot, point sources give a large contribution to the power spectrum at high $\ell$. This can be estimated and removed from the cosmological signal in several different ways. The first technique puts an adjustable constant term in the model $C_{\ell}$, while a second technique fits the difference between frequency bands to a constant $C_{\ell}$. The $\mathrm{CMB}$ gives the same angular power spectrum in different bands, but the contribution of radio point sources is strongly frequency dependent:

$$
C_{\ell}^{\mathbf{i}, \text { src }}=A g_{i} g_{i^{\prime}}\left(\frac{v_{i}}{v_{\mathrm{Q}}}\right)^{\beta}\left(\frac{v_{i^{\prime}}}{v_{\mathrm{Q}}}\right)^{\beta} w_{\ell}^{\mathbf{i}},
$$

where $C_{\ell}^{\mathbf{i}, \text { src }}$ is the point-source contribution to the observed cross-power spectrum between bands $i$ and $i^{\prime}$, the factors $g_{i}$ convert the result to thermodynamic temperature, $v_{\mathrm{Q}} \equiv$ $40.7 \mathrm{GHz}$, and we assume a power-law frequency spectrum with index $\beta=\langle\alpha\rangle-2$. The window function $w_{\ell}^{\mathbf{i}}=b_{\ell}^{i} b_{\ell}^{i^{\prime}} p_{\ell}^{2}$ as in Hinshaw et al. (2007). A third technique computes the effect of unresolved point sources using a model for the counts of sources too faint to be in the catalog. This gives

$$
C_{\ell}=\left(\frac{\partial B_{v}}{\partial T}\right)^{-2} \int_{0}^{S_{\mathrm{lim}}} S^{2}\left(\frac{d N}{d S}\right) d S
$$

for uncorrelated sources, where $\partial B_{v} / \partial T$ converts temperature into intensity, or equivalently the integral of $T d \Omega$ in the definition of $a_{\ell m}$ into flux. Thus the point-source contribution to an observed cross-power spectrum can be written

$$
C_{\ell}^{\mathbf{i}, \mathrm{src}}=\left(\frac{c^{4}}{4 k^{2}\left(v_{i} v_{i^{\prime}}\right)^{2}}\right) g_{i} g_{i^{\prime}} w_{\ell}^{\mathbf{i}} \int S_{i} S_{i^{\prime}} d N
$$

where the integral is over all unmasked sources.

If the wrong spectral index is used to convert the difference between power spectra at different frequencies into a pointsource contribution, then there will be a systematic error in the cosmological parameters, primarily in the spectral index $n_{s}$. This effect can be estimated using a simple model for the correction to the $61 \mathrm{GHz} C_{\ell}$ derived from the difference between the 41 and $94 \mathrm{GHz}$ spectra:

$$
\Delta C_{\ell}^{\mathrm{V}}=v_{V}^{\beta} \frac{C_{\ell}^{\mathrm{Q}}-C_{\ell}^{\mathrm{W}}}{v_{\mathrm{Q}}^{\beta}-v_{\mathrm{W}}^{\beta}} \approx C_{\ell}^{\mathrm{V}}(\beta=-2)(1+0.59(\beta+2)+\ldots) .
$$


Table 2

WMAP Point Source Catalog-Five-Year V-W Bands

\begin{tabular}{|c|c|c|c|c|c|c|c|c|c|}
\hline $\begin{array}{l}\text { R.A. } \\
{[\mathrm{hms}]}\end{array}$ & $\begin{array}{l}\text { Decl. } \\
\text { [dms] }\end{array}$ & $W M A P$ ID & Type & $\begin{array}{c}\text { Dist. } \\
\text { [arcmin] }\end{array}$ & $\begin{array}{l}f_{\mathrm{v}^{\mathrm{a}}} \\
{[\mathrm{Jy}]}\end{array}$ & $\begin{array}{l}T_{\mathrm{V}-\mathrm{W}} \\
{[\mathrm{mK}]} \\
\end{array}$ & $5 \mathrm{GHz}$ ID & $\begin{array}{l}\text { Identified } \\
\text { /Masked }^{\text {b }}\end{array}$ & Note $^{c}$ \\
\hline 000619 & -062731 & WMAP J0006-0623 & $\mathrm{G}$ & 4.1 & $1.7 \pm 0.3$ & 0.35 & PMN J0006-0623 & $\mathrm{Y} / \mathrm{Y}$ & $\ddagger$ \\
\hline 001849 & 732434 & & QSO & 5.0 & $0.9 \pm 0.3$ & 0.23 & GB6 J0019+7327 & $\mathrm{Y} / \mathrm{Y}$ & $\ddagger$ \\
\hline 010843 & 013953 & WMAP J0108+0135 & QSO & 5.0 & $1.8 \pm 0.3$ & 0.36 & PMN J0108+0134 & $\mathrm{Y} / \mathrm{Y}$ & $\ddagger$ \\
\hline 013655 & 475027 & WMAP J0137+4753 & QSO & 1.2 & $3.1 \pm 0.3$ & 0.56 & GB6 J0136+4751 & $\mathrm{Y} / \mathrm{Y}$ & $\ddagger$ \\
\hline 021057 & -510128 & WMAP J0210-5100 & QSO & 1.8 & $2.7 \pm 0.3$ & 0.42 & PMN J0210-5101 & $\mathrm{Y} / \mathrm{Y}$ & 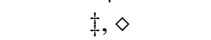 \\
\hline 023750 & 284749 & WMAP $0237+2848$ & QSO & 0.6 & $2.8 \pm 0.3$ & 0.51 & GB6 J0237+2848 & $\mathrm{Y} / \mathrm{Y}$ & $\ddagger$ \\
\hline 031948 & 413013 & WMAP J0319+4131 & G & 0.5 & $5.6 \pm 0.3$ & 1.01 & GB6 J0319+4130 & $\mathrm{Y} / \mathrm{Y}$ & $\ddagger$, Per A \\
\hline 032152 & -370824 & WMAP J0322-3711 & $\mathrm{G}$ & 10.6 & $2.1 \pm 0.3$ & 0.48 & 1Jy 0320-37 & $\mathrm{Y} / \mathrm{Y}$ & $\ddagger$, For A \\
\hline 033419 & -401210 & WMAP J0334-4007 & QSO & 3.9 & $1.6 \pm 0.3$ & 0.24 & PMN J0334-4008 & $\mathrm{Y} / \mathrm{Y}$ & $\ddagger, \diamond$ \\
\hline 035857 & 364006 & $\ldots$ & $\ldots$ & $\ldots$ & 5.0 & 0.79 & $\ldots$ & $\mathrm{N} / \mathrm{Y}$ & In NGC 1499 \\
\hline 040237 & 361710 & $\ldots$ & $\ldots$ & $\ldots$ & 6.2 & 0.99 & 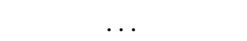 & $\mathrm{N} / \mathrm{Y}$ & In NGC 1499 \\
\hline 040354 & -360448 & WMAP J0403-3604 & QSO & 0.2 & $3.7 \pm 0.3$ & 0.47 & PMN J0403-3605 & $\mathrm{Y} / \mathrm{Y}$ & $\ddagger, \diamond$ \\
\hline 042317 & -012000 & WMAP J0423-0120 & QSO & 0.6 & $7.1 \pm 0.4$ & 1.03 & PMN J0423-0120 & $\mathrm{Y} / \mathrm{Y}$ & $\ddagger$ \\
\hline 042502 & -375642 & WMAP J0424-3757 & QSO & 3.9 & $1.5 \pm 0.3$ & 0.26 & PMN J0424-3756 & $\mathrm{Y} / \mathrm{Y}$ & 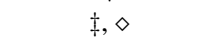 \\
\hline 043321 & 052236 & WMAP J0433+0521 & G & 2.8 & $2.2 \pm 0.3$ & 0.39 & GB6 J0433+0521 & $\mathrm{Y} / \mathrm{Y}$ & $\ddagger$ \\
\hline 044028 & -433455 & WMAP J0440-4332 & QSO & 2.6 & $1.9 \pm 0.3$ & 0.33 & PMN J0440-4332 & $\mathrm{Y} / \mathrm{Y}$ & $\ddagger$ \\
\hline 044914 & 112222 & $\ldots$ & G & 1.8 & $1.9 \pm 0.3$ & 0.34 & GB6 J0449+1121 & $\mathrm{Y} / \mathrm{Y}$ & $*, \dagger, \ddagger$ \\
\hline 045254 & -691856 & $\ldots$ & H II & 5.9 & $1.6 \pm 0.3$ & 0.20 & PMN J0452-6922 & $\mathrm{Y} / \mathrm{N}$ & $\dagger, \ddagger$, in LMC \\
\hline 045543 & -461700 & WMAP 0455-4617 & QSO & 1.7 & $3.4 \pm 0.3$ & 0.60 & PMN J0455-4616 & $\mathrm{Y} / \mathrm{Y}$ & $\ddagger$ \\
\hline 045703 & -662520 & & $\mathrm{H}_{\mathrm{II}}$ & 1.5 & $3.2 \pm 0.3$ & 0.38 & PMN J0456-6624 & $\mathrm{Y} / \mathrm{Y}$ & $\dagger$, in $\mathrm{LMC}$ \\
\hline 045708 & -232428 & WMAP J0456-2322 & QSO & 1.2 & $1.7 \pm 0.3$ & 0.27 & PMN J0457-2324 & $\mathrm{Y} / \mathrm{Y}$ & $\ddagger$ \\
\hline 051752 & -691902 & $\ldots$ & $\mathrm{H}_{\text {II }}$ & 6.8 & $1.0 \pm 0.3$ & 0.19 & PMN J0518-6914 & $\mathrm{Y} / \mathrm{N}$ & $\dagger, \ddagger$, in LMC \\
\hline 051947 & -454821 & WMAP J0519-4546 & $\mathrm{G}$ & 1.7 & $3.5 \pm 0.3$ & 0.63 & 1Jy 0518-45 & $\mathrm{Y} / \mathrm{Y}$ & $\ddagger, \diamond$, Pic A \\
\hline 052219 & -680024 & $\ldots$ & H II & 3.5 & $1.7 \pm 0.3$ & 0.18 & PMN J0522-6757 & $\mathrm{Y} / \mathrm{N}$ & $\dagger, \ddagger$, in LMC \\
\hline 052309 & -362648 & WMAP J0523-3627 & $\mathrm{G}$ & 2.3 & $3.4 \pm 0.3$ & 0.44 & PMN J0522-3628 & $\mathrm{Y} / \mathrm{Y}$ & $\diamond$ \\
\hline 053457 & -673347 & $\ldots$ & $\mathrm{H}_{\text {II }}$ & 3.0 & $1.8 \pm 0.3$ & 0.21 & PMN J0535-6734 & $\mathrm{Y} / \mathrm{N}$ & $\dagger, \ddagger$, in LMC \\
\hline 053517 & -052326 & $\ldots$ & H II & 0.1 & $290.9 \pm 6.7$ & 35.81 & $\ldots$ & $\mathrm{Y} / \mathrm{Y}$ & Ori A \\
\hline 053828 & -690720 & $\ldots$ & $\mathrm{H}_{\mathrm{II}}$ & 3.1 & $26.3 \pm 0.8$ & 3.83 & PMN J0538-6905 & $\mathrm{Y} / \mathrm{Y}$ & $\dagger, \ddagger$, in LMC \\
\hline 053842 & -440549 & WMAP J0538-4405 & QSO & 1.6 & $5.6 \pm 0.3$ & 0.74 & PMN J0538-4405 & $\mathrm{Y} / \mathrm{Y}$ & $\ddagger$ \\
\hline 053853 & -030116 & $\ldots$ & $\ldots$ & $\ldots$ & 1.9 & 0.31 & $\ldots$ & $\mathrm{N} / \mathrm{N}$ & in Ori \\
\hline 054010 & -030851 & $\ldots$ & $\ldots$ & $\ldots$ & 2.0 & 0.32 & $\ldots$ & $\mathrm{N} / \mathrm{N}$ & in Ori \\
\hline 054030 & -023903 & $\ldots$ & $\ldots$ & $\ldots$ & 2.6 & 0.41 & $\ldots$ & $\mathrm{N} / \mathrm{N}$ & in Ori \\
\hline 054143 & -015349 & $\ldots$ & $\mathrm{H}$ II & 0.2 & $45.8 \pm 1.2$ & 5.78 & PMN J0541-0154 & $\mathrm{Y} / \mathrm{Y}$ & $\ddagger$, Ori B \\
\hline 060758 & -062625 & $\ldots$ & $\mathrm{H}$ II & 4.5 & $7.0 \pm 0.4$ & 0.37 & PMN J0607-0623 & $\mathrm{Y} / \mathrm{Y}$ & $\dagger, *, \ddagger$ \\
\hline 060938 & -154135 & WMAP J0609-1541 & QSO & 1.3 & $2.2 \pm 0.3$ & 0.37 & PMN J0609-1542 & $\mathrm{Y} / \mathrm{Y}$ & $\ddagger, \diamond$ \\
\hline 063536 & -751515 & WMAP J0635-7517 & QSO & 1.2 & $2.5 \pm 0.3$ & 0.42 & PMN J0635-7516 & $\mathrm{Y} / \mathrm{Y}$ & $\ddagger \diamond \diamond$ \\
\hline 072154 & -373036 & $\ldots$ & $\ldots$ & $\ldots$ & 1.6 & 0.25 & & $\mathrm{~N} / \mathrm{N}$ & in Gum \\
\hline 072240 & 712053 & WMAP J0721+7122 & QSO & 3.7 & $1.9 \pm 0.3$ & 0.25 & GB6 J0721+7120 & $\mathrm{Y} / \mathrm{Y}$ & $\ddagger$ \\
\hline 073122 & -480924 & $\ldots$ & $\ldots$ & $\ldots$ & 1.7 & 0.26 & $\ldots$ & $\mathrm{N} / \mathrm{N}$ & in Gum \\
\hline 073458 & -484911 & $\ldots$ & $\ldots$ & $\ldots$ & 2.1 & 0.33 & $\ldots$ & $\mathrm{N} / \mathrm{N}$ & in Gum \\
\hline 073627 & -495052 & $\cdots$ & $\cdots$ & $\cdots$ & 1.5 & 0.24 & $\cdots$ & $\mathrm{N} / \mathrm{N}$ & in Gum \\
\hline 074433 & -503349 & $\ldots$ & $\ldots$ & $\ldots$ & 1.6 & 0.25 & $\ldots$ & $\mathrm{N} / \mathrm{N}$ & in Gum \\
\hline 074903 & -503905 & $\ldots$ & $\ldots$ & $\ldots$ & 1.6 & 0.26 & $\ldots$ & $\mathrm{N} / \mathrm{N}$ & in Gum \\
\hline 080232 & -504125 & $\ldots$ & $\ldots$ & $\ldots$ & 1.6 & 0.26 & $\ldots$ & $\mathrm{N} / \mathrm{N}$ & in Gum \\
\hline 081406 & -525202 & $\ldots$ & $\ldots$ & $\ldots$ & 1.6 & 0.25 & $\ldots$ & $\mathrm{N} / \mathrm{N}$ & in Gum \\
\hline 083638 & -201552 & WMAP J0836-2015 & QSO & 1.2 & $1.9 \pm 0.3$ & 0.39 & PMN J0836-2017 & $\mathrm{Y} / \mathrm{Y}$ & $\ddagger$ \\
\hline 084121 & 705529 & WMAP J0841+7053 & QSO & 1.8 & $1.7 \pm 0.3$ & 0.31 & GB6 J0841+7053 & $\mathrm{Y} / \mathrm{Y}$ & $\ddagger$ \\
\hline 085458 & 200606 & WMAP J0854+2006 & QSO & 2.2 & $4.2 \pm 0.3$ & 0.61 & GB6 J0854+2006 & $\mathrm{Y} / \mathrm{Y}$ & $\ddagger$ \\
\hline 090912 & 012337 & WMAP J0909+0119 & QSO & 2.1 & $1.6 \pm 0.3$ & 0.44 & GB6 J0909+0121 & $\mathrm{Y} / \mathrm{Y}$ & $\ddagger$ \\
\hline 092128 & 072422 & $\ldots$ & $\ldots$ & $\ldots$ & 2.3 & 0.36 & (2) & $\mathrm{N} / \mathrm{N}$ & $\ldots$ \\
\hline 092705 & 390346 & WMAP J0927+3901 & QSO & 1.5 & $4.6 \pm 0.3$ & 0.72 & GB6 J0927+3902 & $\mathrm{Y} / \mathrm{Y}$ & $\ddagger$ \\
\hline 105831 & 013344 & WMAP J1058+0134 & QSO & 0.4 & $4.2 \pm 0.3$ & 0.80 & GB6 J1058+0133 & $\mathrm{Y} / \mathrm{Y}$ & $\ddagger$ \\
\hline 105909 & -800412 & WMAP J1059-8003 & QSO & 1.1 & $2.3 \pm 0.3$ & 0.30 & PMN J1058-8003 & $\mathrm{Y} / \mathrm{Y}$ & 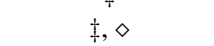 \\
\hline 115304 & 492955 & WMAP J1153+4932 & G & 3.5 & $2.1 \pm 0.3$ & 0.30 & GB6 J1153+4931 & $\mathrm{Y} / \mathrm{Y}$ & $\ldots$ \\
\hline 115941 & 291949 & WMAP J1159+2915 & QSO & 5.5 & $1.8 \pm 0.3$ & 0.33 & GB6 J1159+2914 & $\mathrm{Y} / \mathrm{Y}$ & $\ddagger$ \\
\hline 122908 & 020306 & WMAP J1229+0203 & QSO & 0.3 & $14.6 \pm 0.5$ & 2.32 & PMN J1229+0203 & $\mathrm{Y} / \mathrm{Y}$ & $\ddagger$ \\
\hline 123049 & 122256 & WMAP J1230+1223 & G & 0.5 & $9.6 \pm 0.4$ & 1.49 & GB6 J1230+1223 & $\mathrm{Y} / \mathrm{Y}$ & Vir A \\
\hline 124702 & -254632 & WMAP J1246-2547 & QSO & 3.7 & $1.7 \pm 0.3$ & 0.33 & PMN J1246-2547 & $\mathrm{Y} / \mathrm{Y}$ & $\ddagger$ \\
\hline 125612 & -054728 & WMAP J1256-0547 & QSO & 0.2 & $16.9 \pm 0.6$ & 2.45 & PMN J1256-0547 & $\mathrm{Y} / \mathrm{Y}$ & $\ddagger$ \\
\hline 131047 & 322425 & WMAP J1310+3222 & QSO & 5.3 & $1.7 \pm 0.3$ & 0.28 & GB6 J1310+3220 & $\mathrm{Y} / \mathrm{Y}$ & $\ddagger$ \\
\hline 131559 & -334518 & WMAP J1316-3337 & QSO & 6.6 & $1.9 \pm 0.3$ & 0.33 & PMN J1316-3339 & $\mathrm{Y} / \mathrm{Y}$ & $\dagger, \diamond$ \\
\hline 132235 & -443825 & $\ldots$ & $\ldots$ & $\ldots$ & 2.2 & 0.34 & 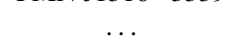 & $\mathrm{N} / \mathrm{Y}$ & In Cen A vicinity \\
\hline 132533 & -425925 & $\ldots$ & $\mathrm{G}$ & 2.0 & $25.6 \pm 0.8$ & 4.02 & PMN J1325-4257 & $\mathrm{Y} / \mathrm{Y}$ & $\dagger, *, \diamond$, Cen A \\
\hline 133741 & -125637 & WMAP J1337-1257 & QSO & 0.8 & $6.0 \pm 0.3$ & 0.86 & PMN J1337-1257 & $\mathrm{Y} / \mathrm{Y}$ & $\ddagger$ \\
\hline 142753 & -420636 & WMAP J1427-4206 & QSO & 0.7 & $2.7 \pm 0.3$ & 0.39 & PMN J1427-4206 & $\mathrm{Y} / \mathrm{Y}$ & $\dagger, \diamond$ \\
\hline
\end{tabular}


Table 2

(Continued)

\begin{tabular}{|c|c|c|c|c|c|c|c|c|c|}
\hline $\begin{array}{l}\text { R.A. } \\
{[\mathrm{hms}]}\end{array}$ & $\begin{array}{l}\text { Decl. } \\
\text { [dms] }\end{array}$ & $W M A P$ ID & Type & $\begin{array}{c}\text { Dist. } \\
\text { [arcmin] }\end{array}$ & $\begin{array}{l}f_{\mathrm{V}^{\mathrm{a}}} \\
{[\mathrm{Jy}]}\end{array}$ & $\begin{array}{l}T_{\mathrm{V}-\mathrm{W}} \\
{[\mathrm{mK}]} \\
\end{array}$ & $5 \mathrm{GHz}$ ID & $\begin{array}{l}\text { Identified } \\
\text { /Masked }^{\text {b }}\end{array}$ & Note $^{\mathrm{c}}$ \\
\hline 151804 & -242126 & WMAP J1517-2421 & $\mathrm{G}$ & 5.1 & $1.8 \pm 0.3$ & 0.34 & PMN J1517-2422 & $\mathrm{Y} / \mathrm{Y}$ & $\ddagger, \diamond$ \\
\hline 154933 & 023346 & WMAP J1549+0236 & QSO & 3.4 & $2.1 \pm 0.3$ & 0.36 & GB6 J1549+0237 & $\mathrm{Y} / \mathrm{Y}$ & $\ddagger$ \\
\hline 161342 & 341203 & WMAP J1613+3412 & QSO & 0.8 & $2.9 \pm 0.3$ & 0.51 & GB6 J1613+3412 & $\mathrm{Y} / \mathrm{Y}$ & $\ddagger$ \\
\hline 161832 & -771231 & WMAP J1618-7716 & QSO & 5.3 & $1.7 \pm 0.3$ & 0.25 & PMN J1617-7717 & $\mathrm{Y} / \mathrm{Y}$ & $\ddagger, \diamond$ \\
\hline 162018 & -253447 & $\ldots$ & $\ldots$ & $\ldots$ & 2.7 & 0.43 & $\ldots$ & $\mathrm{N} / \mathrm{N}$ & in Oph \\
\hline 162051 & -252111 & $\ldots$ & $\ldots$ & $\ldots$ & 3.0 & 0.47 & $\ldots$ & $\mathrm{N} / \mathrm{N}$ & in Oph \\
\hline 162838 & -090359 & $\ldots$ & $\ldots$ & $\ldots$ & 2.1 & 0.34 & $\ldots$ & $\mathrm{N} / \mathrm{N}$ & in Oph \\
\hline 163505 & 380811 & WMAP J1635+3807 & QSO & 2.1 & $4.1 \pm 0.3$ & 0.56 & GB6 J1635+3808 & $\mathrm{Y} / \mathrm{Y}$ & $\ddagger$ \\
\hline 163826 & 571839 & WMAP J1638+5722 & QSO & 2.4 & $1.6 \pm 0.3$ & 0.26 & GB6 J1638+5720 & $\mathrm{Y} / \mathrm{Y}$ & $\ddagger$ \\
\hline 164226 & 685554 & WMAP J1642+6854 & QSO & 1.8 & $1.3 \pm 0.3$ & 0.31 & GB6 J1642+6856 & $\mathrm{Y} / \mathrm{Y}$ & $\ddagger$ \\
\hline 164256 & 394844 & WMAP J1642+3948 & QSO & 0.6 & $5.1 \pm 0.3$ & 0.78 & GB6 J1642+3948 & $\mathrm{Y} / \mathrm{Y}$ & $\ddagger$ \\
\hline 172032 & -005959 & $\ldots$ & G & 1.5 & $2.6 \pm 0.3$ & 0.43 & PMN J1720-0058 & $\mathrm{Y} / \mathrm{Y}$ & $\dagger, *$ \\
\hline 173301 & -130625 & $\ldots$ & QSO & 1.6 & $3.3 \pm 0.3$ & 0.51 & PMN J1733-1304 & $\mathrm{Y} / \mathrm{Y}$ & $*, \ddagger$ \\
\hline 174356 & -034842 & $\ldots$ & QSO & 1.6 & $4.5 \pm 0.3$ & 0.76 & PMN J1743-0350 & $\mathrm{Y} / \mathrm{Y}$ & $*, \ddagger$ \\
\hline 175138 & 094023 & $\ldots$ & QSO & 1.9 & $3.6 \pm 0.3$ & 0.57 & GB6 J1751+0938 & $\mathrm{Y} / \mathrm{Y}$ & $\dagger, *, \ddagger$ \\
\hline 175344 & 284737 & WMAP J1753+2848 & QSO & 0.6 & $2.0 \pm 0.3$ & 0.27 & GB6 J1753+2847 & $\mathrm{Y} / \mathrm{Y}$ & $\ddagger$ \\
\hline 180649 & 695036 & WMAP J1806+6949 & $\mathrm{G}$ & 1.1 & $1.3 \pm 0.3$ & 0.23 & GB6 J1806+6949 & $\mathrm{Y} / \mathrm{Y}$ & $\ddagger$ \\
\hline 182937 & 484645 & WMAP J1829+4845 & QSO & 2.2 & $2.3 \pm 0.3$ & 0.28 & GB6 J1829+4844 & $\mathrm{Y} / \mathrm{Y}$ & $\ldots$ \\
\hline 192452 & -291415 & $\ldots$ & QSO & 0.3 & $10.1 \pm 0.4$ & 1.60 & PMN J1924-2914 & $\mathrm{Y} / \mathrm{Y}$ & $\dagger, *, \ddagger, \diamond$ \\
\hline 192742 & 735634 & WMAP J1927+7357 & QSO & 1.5 & $3.0 \pm 0.3$ & 0.45 & GB6 J1927+7357 & $\mathrm{Y} / \mathrm{Y}$ & $\ddagger$ \\
\hline 195755 & -384503 & WMAP J1958-3845 & QSO & 0.9 & $2.9 \pm 0.3$ & 0.35 & PMN J1957-3845 & $\mathrm{Y} / \mathrm{Y}$ & $\ddagger, \diamond$ \\
\hline 201112 & -154751 & WMAP J2011-1547 & QSO & 1.5 & $1.1 \pm 0.3$ & 0.32 & PMN J2011-1546 & $\mathrm{Y} / \mathrm{Y}$ & $\ddagger, \diamond$ \\
\hline 205601 & -471556 & WMAP J2056-4716 & QSO & 2.8 & $2.6 \pm 0.3$ & 0.39 & PMN J2056-4714 & $\mathrm{Y} / \mathrm{Y}$ & 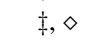 \\
\hline 213415 & -015935 & WMAP J2134-0154 & QSO & 6.4 & $1.3 \pm 0.3$ & 0.38 & PMN J2134-0153 & $\mathrm{Y} / \mathrm{Y}$ & $\ddagger$ \\
\hline 214807 & 065629 & WMAP J2148+0657 & QSO & 1.2 & $6.5 \pm 0.3$ & 1.02 & GB6 J2148+0657 & $\mathrm{Y} / \mathrm{Y}$ & $\ddagger$ \\
\hline 215719 & -694142 & WMAP J2157-6942 & $\mathrm{G}$ & 1.2 & $2.1 \pm 0.3$ & 0.41 & PMN J2157-6941 & $\mathrm{Y} / \mathrm{Y}$ & $\diamond$ \\
\hline 220247 & 421737 & WMAP J2202+4217 & QSO & 1.2 & $3.6 \pm 0.3$ & 0.39 & GB6 J2202+4216 & $\mathrm{Y} / \mathrm{Y}$ & $\ldots$ \\
\hline 221855 & -033048 & WMAP J2218-0335 & QSO & 4.9 & $1.5 \pm 0.3$ & 0.35 & PMN J2218-0335 & $\mathrm{Y} / \mathrm{Y}$ & $\ddagger$ \\
\hline 222542 & -045756 & WMAP J2225-0455 & QSO & 1.6 & $3.6 \pm 0.3$ & 0.68 & PMN J2225-0457 & $\mathrm{Y} / \mathrm{Y}$ & $\ddagger$ \\
\hline 222946 & -083123 & WMAP J2229-0833 & QSO & 2.1 & $2.8 \pm 0.3$ & 0.41 & PMN J2229-0832 & $\mathrm{Y} / \mathrm{Y}$ & $\ddagger$ \\
\hline 223235 & 114250 & WMAP J2232+1144 & QSO & 1.1 & $4.2 \pm 0.3$ & 0.53 & GB6 J2232+1143 & $\mathrm{Y} / \mathrm{Y}$ & $\ldots$ \\
\hline 223521 & -483513 & WMAP J2235-4834 & QSO & 1.5 & $2.1 \pm 0.3$ & 0.36 & PMN J2235-4835 & $\mathrm{Y} / \mathrm{Y}$ & $\ddagger, \diamond$ \\
\hline 225357 & 161011 & WMAP J2254+1608 & QSO & 1.3 & $8.0 \pm 0.4$ & 1.06 & GB6 J2253+1608 & $\mathrm{Y} / \mathrm{Y}$ & $\ddagger$ \\
\hline 225810 & -275931 & WMAP J2258-2757 & QSO & 1.5 & $4.7 \pm 0.3$ & 0.75 & PMN J2258-2758 & $\mathrm{Y} / \mathrm{Y}$ & $\ddagger \diamond \diamond$ \\
\hline
\end{tabular}

Notes.

a The V-band fluxes of the identified sources are calculated as in Chen \& Wright (2008); The fluxes of the unidentified sources are estimated by multiplying the $\mathrm{V}-\mathrm{W}$ temperature in the filtered map with the median conversion factor from V-W temperatures to V-band fluxes of the identified sources and are given without an uncertainty.

b Three-year WMAP point source mask is considered here.

${ }^{\mathrm{c}} \dagger$ and $*$ indicate the new sources cross-detected in Nie \& Zhang (2007) and López-Caniego et al. (2007). $\ddagger$ and $\diamond$ indicate the source is included in the CRATES catalog (Healey et al. 2007) and the AT20G BSS catalog (Massardi et al. 2007), respectively.

${ }^{\mathrm{d}}$ Indicates the source has multiple possible $5 \mathrm{GHz}$ identifications. The brightest one is given here.

Table 3

Significant Polarization Percentages

\begin{tabular}{lccc}
\hline \hline WMAP ID & $\mathrm{K}$ & $\mathrm{Ka}$ & $\mathrm{Q}$ \\
\hline J0322-3711 & $8.5 \pm 0.4$ & $9.3 \pm 1.2$ & $8.1 \pm 2.2$ \\
J0519-4546 & $5.7 \pm 0.9$ & $8.6 \pm 2.0$ & $\ldots$ \\
J1229+0203 & $4.9 \pm 0.3$ & $4.4 \pm 0.6$ & $4.8 \pm 0.8$ \\
J1230+1223 & $3.8 \pm 04$ & $4.8 \pm 0.7$ & $4.4 \pm 1.0$ \\
J1256-0547 & $3.6 \pm 0.4$ & $3.1 \pm 0.7$ & $3.8 \pm 0.8$ \\
\hline
\end{tabular}

Thus if $\beta$ were really -2.09 instead of -2 then the correction to the $61 \mathrm{GHz}$ power spectrum should be $5 \%$ smaller than that which would be estimated assuming $\beta=-2$. Huffenberger et al. (2006) found that decreasing the point-source correction by $44 \%$ changed the spectral index $n_{s}$ by 0.018 so changing $\beta$ from -2.0 to -2.09 would change $n_{s}$ by 0.0022 , or $0.15 \sigma$.

\section{SUMMARY AND CONCLUSIONS}

There are no other radio surveys that provide the wide coverage of WMAP at frequencies from 23-100 GHz. In addition, $W M A P$ provides year-by-year fluxes to track the variability of bright millimeter-wave sources. We present catalogs of point sources found in the WMAP five-year dataset. Two different approaches have been used: the standard approach of looking for peaks in single band maps that have been convolved with a matched filter, and a new approach that constructs CMB-free internal linear combination maps. Using the 61 and $94 \mathrm{GHz}$ data gives a catalog with somewhat lower sensitivity than the standard approach, but with better positional accuracy. The estimated contamination of the CMB angular power spectrum by unmasked point sources has been estimated, with results that are consistent with previous analyses and with the differences between angular power spectra in different bands (Nolta et al. 2009). Remaining uncertainties in the point-source correction 


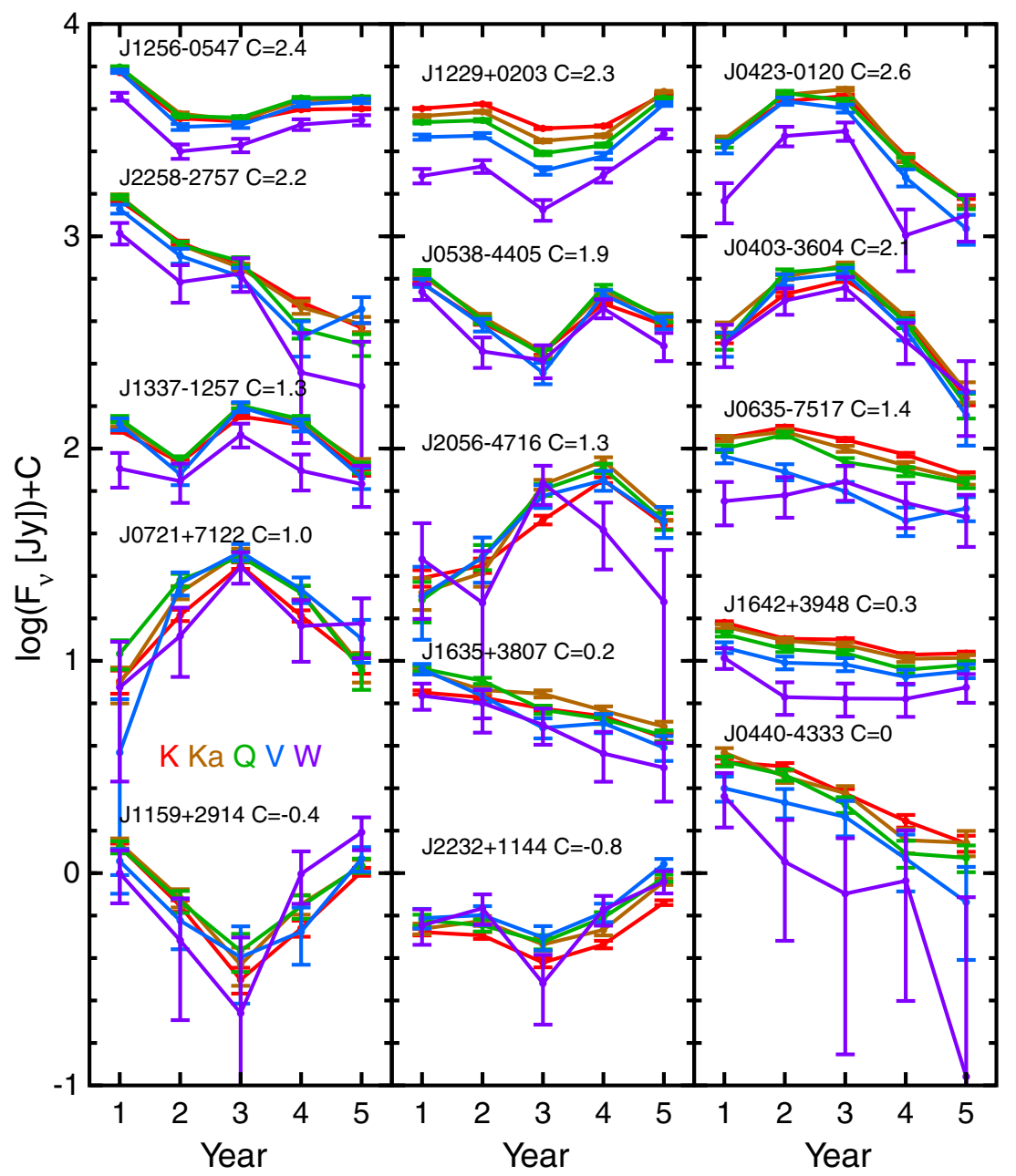

Figure 4. 15 sources with the highest $\chi^{2}$ for a fit of a constant flux with an arbitrary spectrum. The $23 \mathrm{GHz}$ data are plotted in red, the $33 \mathrm{GHz}$ data are plotted in orange, the $41 \mathrm{GHz}$ data are plotted in green, the $61 \mathrm{GHZ}$ data are plotted in blue, and the $94 \mathrm{GHz}$ data are plotted in purple.

contribute to the uncertainty of the cosmological parameters, with the biggest effect occurring for $n_{s}$.

The WMAP mission is made possible by the support of the Science Mission Directorate Office at NASA Headquarters. This research was additionally supported by NASA grants NNG05GE76G, NNX07AL75G S01, LTSA03-0000090, ATPNNG04GK55G, and ADP03-0000-092. E.K. acknowledges support from an Alfred P. Sloan Research Fellowship. This research has made use of NASA's Astrophysics Data System Bibliographic Services. We acknowledge use of the HEALPix, CAMB, and CMBFAST packages.

\section{REFERENCES}

Bennett, C. L., et al. 2003a, ApJ, 583, 1

Bennett, C. L., et al. 2003b, ApJS, 148, 97

Chen, X., \& Wright, E. 2008, ApJ, 681, 747

Cleary, K. A., et al. 2005, MNRAS, 360, 340

de Zotti, G., Ricci, R., Mesa, D., Silva, L., Mazzotta, P., Toffolatti, L., \&

González-Nuevo, J. 2005, A\&A, 431, 893

Eddington, A. S. 1913, MNRAS, 73, 359

Giommi, P., et al. 2007, A\&A, 468, 571
Gregory, P. C., Scott, W. K., Douglas, K., \& Condon, J. J. 1996, ApJS, 103, 427 Griffith, M. R., Wright, A. E., Burke, B. F., \& Ekers, R. D. 1994, ApJS, 90, 179

Healey, S. E., Romani, R. W., Taylor, G. B., Sadler, E. M., Ricci, R., Murphy, T., Ulvestad, J. S., \& Winn, J. N. 2007, ApJS, 171, 61

Hill, R., et al. 2009, ApJS, 180, 246

Hinshow, G., et al. 2007, ApJS, 170, 288

Huffenberger, K. M., Eriksen, H. K., \& Hansen, F. K. 2006, ApJ, 651, L81

Jarosik, N., et al. 2007, ApJS, 170, 263

Kovac, J. M., Leitch, E. M., Pryke, C., Carlstrom, J. E., Halverson, N. W., \& Holzapfel, W. L. 2002, Nature, 420, 772

Kühr, H., Witzel, A., Pauliny-Toth, I. I. K., \& Nauber, U. 1981, A\&AS, 45, 367

López-Caniego, M., González-Nuevo, J., Herranz, D., Massardi, M., Sanz, J. L., De Zotti, G., Toffolatti, L., \& Argüeso, F. 2007, ApJS, 170, 108 Mason, B. S., et al. 2003, ApJ, 591, 540

Massardi, M., et al. 2007, arXiv:0709.3485

Nie, J.-Y., \& Zhang, S.-N. 2007, Chin. J. Astron. Astrophys., 7, 199

Nolta, M. R., et al. 2009, ApJS, 180, 296

Page, L., et al. 2003, ApJS, 148, 39

Refregier, A., Spergel, D. N., \& Herbig, T. 2000, ApJ, 531, 31

Spergel, D. N., et al. 2007, ApJS, 170, 377

Tegmark, M., \& de Oliveira-Costa, A. 1998, ApJ, 500, L83

Toffolatti, L., Argueso Gomez, F., de Zotti, G., Mazzei, P., Franceschini, A., Danese, L., \& Burigana, C. 1998, MNRAS, 297, 117

Trushkin, S. A. 2003, Bull. Spec. Astrophys. Obs. N. Caucasus, 55, 90 